\title{
Dynamically assisted Schwinger mechanism and chirality production in parallel electromagnetic field
}

\author{
Hidetoshi Taya ${ }^{*} *$ \\ Department of Physics and Center for Field Theory and Particle Physics, Fudan University, Shanghai 200433, China
}

(Received 26 March 2020; accepted 8 May 2020; published 1 June 2020)

\begin{abstract}
We study particle and chirality production from the vacuum in the presence of a slow strong parallel electromagnetic field superimposed by a fast weak perturbative electromagnetic field. We derive an analytical formula for the particle and chirality production number based on the perturbation theory in the Furry picture. With the formula, we analytically discuss the interplay and dynamical assistance between the Schwinger mechanism and one-photon pair production and clarify effects of a parallel slow strong magnetic field. We also show that the dynamical assistance can significantly enhance chirality production, and that a sizable amount of chirality can be produced even for massive particles. Phenomenological applications including heavy-ion collisions and intense laser experiments are also discussed.
\end{abstract}

DOI: 10.1103/PhysRevResearch.2.023257

\section{INTRODUCTION}

One of the most remarkable predictions of quantum electrodynamics (QED) is spontaneous particle production from the vacuum in the presence of a slow strong electromagnetic field (for review, see Refs. [1-3]). This remarkable prediction was first made by Sauter in 1931 [4]. Sauter's idea was expanded later by Heisenberg and Euler [5] and by Schwinger [6], who fully formulated the idea within quantum field theory for the first time. Thus, the vacuum particle production by a slow strong electromagnetic field is called the Schwinger mechanism. The Schwinger mechanism is essentially an electric effect. In the presence of a slow strong electric field, there occurs a level crossing between the Dirac sea and the positive energy continuum (see Fig. 1). Then, an electron filling the Dirac sea can tunnel into the positive energy continuum, leaving a hole in the Dirac sea. Thus, a pair of an electron and a positron is spontaneously produced, which can be understood as a QED analog of electrical breakdown or the Landau-Zener transition in materials [7-10].

The production number of the Schwinger mechanism is determined by the tunneling rate, which decreases with increasing the gap size $\sim m$ and the tunneling length $\sim m / e \bar{E}$ (see Fig. 1), where $m, e>0$, and $\bar{E}$ are mass, the QED coupling constant, and the electric field strength, respectively. Therefore, one may expect that the production number is suppressed by an exponential of $m \times m / e \bar{E}$. Indeed, Schwinger [6] showed that the production number of electrons $N$ and positrons $\bar{N}$ for a constant and homogeneous electric

\footnotetext{
*h_taya@fudan.edu.cn

Published by the American Physical Society under the terms of the Creative Commons Attribution 4.0 International license. Further distribution of this work must maintain attribution to the author(s) and the published article's title, journal citation, and DOI.
}

field is given by

$$
N=\bar{N}=V T \times \frac{(e \bar{E})^{2}}{4 \pi^{3}} e^{-\pi \frac{m^{2}}{e \bar{E}}}
$$

with $V$ and $T$ being the spatial volume and the whole time interval, respectively. For a more general electromagnetic field configuration, the production number formula reads [6,11-17] (see also Refs. [18-24] for more discussions on magnetic-field effects)

$$
N=\bar{N}=V T \times \frac{(e \bar{E})^{2}}{4 \pi^{3}} e^{-\pi \frac{m^{2}}{e \bar{E}}} \times \pi \frac{e \bar{B}}{e \bar{E}} \operatorname{coth}\left[\pi \frac{e \bar{B}}{e \bar{E}}\right] .
$$

Remark that it is sufficient to consider a parallel electromagnetic field configuration $\overline{\boldsymbol{E}} \| \overline{\boldsymbol{B}}$, which can cover all the possible values of the Lorentz invariants $\mathcal{F} \equiv \overline{\boldsymbol{E}}^{2}-\overline{\boldsymbol{B}}^{2}$ and $\mathcal{G} \equiv \overline{\boldsymbol{E}} \cdot \overline{\boldsymbol{B}}$. Equation (2) shows that the addition of a parallel magnetic field enhances the production number by the factor $\pi(e \bar{B} / e \bar{E}) \operatorname{coth}[\pi(e \bar{B} / e \bar{E})]>1 .^{1}$ The Landau quantization is the essence of this enhancement. Namely, the Landau quantization discretizes the transverse mass $\sqrt{m^{2}+p_{\perp}^{2}} \rightarrow$ $\sqrt{m^{2}+|e \bar{B}|(2 n+1-s)}$ (where $n=0,1,2, \ldots \in \mathbb{N}$ labels the Landau level, and $s= \pm 1$ is the spin component with respect to the magnetic field direction) and, accordingly, the phase space $\int d^{2} \boldsymbol{p}_{\perp} \rightarrow e \bar{B} \sum_{n}$. The phase space linearly increases with $e \bar{B}$, which essentially accounts for the enhancement of the production number of electrons and positrons. The

\footnotetext{
${ }^{1}$ Precisely speaking, a parallel magnetic field enhances the production number except for scalar particles, whose lowest energy level increases with $e \bar{B}$ as $\sqrt{m^{2}+|e \bar{B}|}$ and hence its production is exponentially suppressed $[17,18]$. The production of vector particles (e.g., gluon and $W$ boson) or, more generally, higher spin particles, is enhanced more strongly than that of spinor particles [12,24] because of the existence of unstable modes (Nielsen-Olesen instability [25-27]).
} 


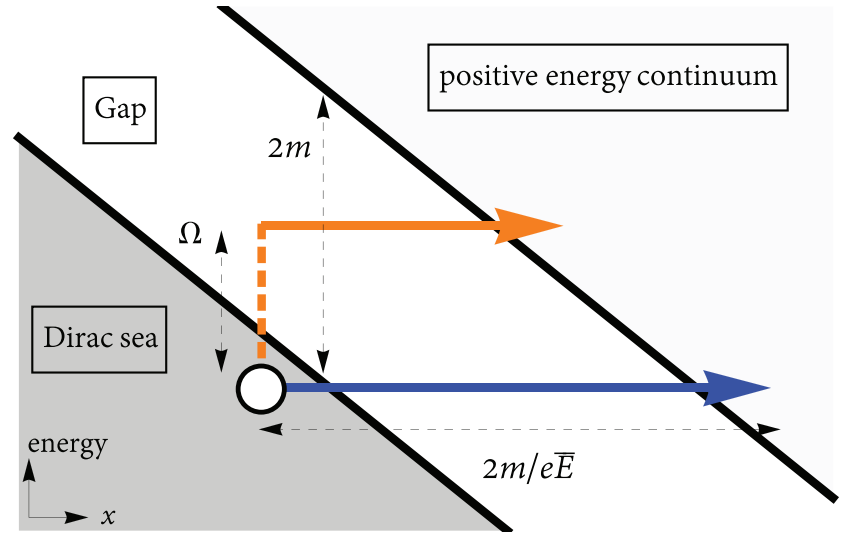

FIG. 1. Band structure of the QED vacuum in the presence of a slow strong electric field applied in the $x$ direction, $V(x)=-e \bar{E} x$, and schematic pictures of the Schwinger mechanism [blue (darker gray in grayscale) arrow] and the dynamically assisted Schwinger mechanism [orange (lighter gray in grayscale) arrow]. $2 m$, with $m$ being mass, is the gap size, and $\Omega$ represents energy supplied by a fast weak electromagnetic field in the dynamically assisted Schwinger mechanism.

mass suppression factor $\exp \left[-\pi m^{2} / e \bar{E}\right]$ remains the same since the gap size $\sim m$ and the tunneling length $\sim m / e \bar{E}$ are unchanged by the Landau quantization, or the presence of a magnetic field, for spinor particles.

The Schwinger mechanism (or the idea of the Schwinger mechanism) has a wide spectrum of phenomenological applications including, to name a few, the early Universe [28-34], condensed matter/materials [35-43], and axion production [44-46]. In particular, application to heavy-ion collisions has received much attention over the decades. Just after a collision of heavy ions at the Relativistic Heavy Ion Collider (RHIC) and the Large Hadron Collider (LHC), a strong (chromo-electromagnetic field, which is sometimes called glamsa, is produced [47-51]. It is widely recognized that particle production from the glasma via the Schwinger mechanism is the essence of the formation of the quark-gluon plasma in heavy-ion collisions [17,50-55], while its detailed understanding is still lacking. In addition, the glasma has a parallel (chromo)electromagnetic field configuration, and therefore produces chirality through the chiral anomaly [56-58]. The chirality imbalance may induce novel anomalous transport phenomena (see Refs. [59,60] for review) such as the chiral magnetic effect [61-63]. Experimental search for anomalous transport phenomena is a very active topic in heavyion collision experiments; see Ref. [64] for a recent review. Therefore, it is important to deepen our understanding of particle and chirality production in the presence of a parallel strong electromagnetic field via the Schwinger mechanism and discuss possible observable consequences in, e.g., heavyion collisions.

The Schwinger mechanism has not been observed in laboratory experiments yet, despite its great theoretical/phenomenological interest. The most promising way to observe the Schwinger mechanism in laboratory experiments is to use intense lasers. However, the Schwinger mechanism is exponentially suppressed by the mass factor as in Eqs. (1) and (2), and hence an electric field strength of the order of the electron mass scale $e \bar{E}_{\mathrm{cr}} \equiv m_{e}^{2} \sim \sqrt{10^{29} \mathrm{~W} / \mathrm{cm}^{2}}$ is required for the Schwinger mechanism to be manifest. The available field strength at the present is limited to $e \bar{E} \sim \sqrt{10^{22} \mathrm{~W} / \mathrm{cm}^{2}}$ [65]. Upcoming intense laser facilities such as the Extreme Light Infrastructure (ELI) and at the Exawatt Center for Extreme Light Studies (XCELS) are expected to reach $e \bar{E} \sim \sqrt{10^{24-25} \mathrm{~W} / \mathrm{cm}^{2}}$ but are still weaker than the critical field strength $\bar{E}_{\text {cr }}$ by several orders of magnitude (see Ref. [66] for a review of the current experimental situation). Therefore, it is still difficult within the current laser technology to directly observe the Schwinger mechanism.

The experimental difficulty motivated theorists to investigate how to enhance the Schwinger mechanism to observe it with a subcritical electromagnetic field. One of the promising ideas is the dynamically assisted Schwinger mechanism [67-71], which is an analog of the Franz-Keldysh effect in semiconductor physics [72-75]. The idea is to superimpose a weak fast electromagnetic field with a large Keldysh parameter [18,76-78] (or inject an energetic dynamical photon) onto a slow strong electromagnetic field (see Fig. 1). Then, the weak fact electromagnetic field perturbatively interacts with electrons in the Dirac sea and supplies energy, which is depicted by the orange (lighter gray in grayscale) dashed line in Fig. 1. The electrons come out from the Dirac sea, and the gap size as well as the tunneling length are effectively reduced, which implies a reduction of the mass suppression factor in the production number formulas (1) and (2). Therefore, the Schwinger mechanism is enhanced, and the enhancement was found to be significant [67-71], providing a hope to observe the Schwinger mechanism, albeit indirectly, with intense lasers in the near future.

A number of aspects of the dynamically assisted Schwinger mechanism have been investigated/clarified within, for example, the worldline instanton formalism [79-81], numerical simulations based on the quantum kinetic theory $[82,83]$, and the recently developed perturbation theory in the Furry picture [74,75,84-89]. Examples include optimization of a field profile [90-95], momentum distribution [74,75,87,88,96-102], finite-size effects [103,104], and spatially dependent perturbations [105-108]. In particular, it was clarified in Refs. [74,75] that the dynamically assisted Schwinger mechanism can be understood in terms of the interplay between the Schwinger mechanism and one-photon pair production process $\gamma+\bar{E} \rightarrow e^{+} e^{-}$. Namely, in the presence of both a slow strong electromagnetic field and a fast weak perturbation, the Schwinger mechanism and one-photon pair production dominate the particle production if the frequencies (i.e., the energy supply) of the perturbation are small and large, respectively. At intermediate frequencies, both production mechanisms take place and assist each other to significantly enhance the particle production; i.e., the dynamically assisted Schwinger mechanism occurs.

Although there are many preceding studies on the dynamically assisted Schwinger mechanism, they focus mostly on a slow strong purely electric field configuration and, therefore, effects of a parallel strong magnetic field are less understood 
(effects of a transverse strong magnetic field associated with a finite-sized strong electric field were recently discussed in Ref. [109]). In other words, the dynamically assisted Schwinger mechanism for a nonvanishing Lorentz invariant $\mathcal{G} \neq 0$ is unexplored, and it is unclear how the dynamical assistance affects chirality production. These questions are important to complete our understanding of the dynamically assisted Schwinger mechanism as well as to discuss phenomenology such as anomalous transport phenomena in heavy-ion collisions and to propose new observables in the upcoming laser experiments. To the best of our knowledge, Ref. [106] is the only work in this direction at the present. Reference [106] investigated the dynamically assisted Schwinger mechanism in the presence of a parallel slow strong electromagnetic field configuration within the worldline instanton formalism just for a slowly varying perturbation (only for which the worldline instanton formalism can be justified) with Sauter-type spacetime dependence, and chirality production was not discussed explicitly. It is important to go beyond a slowly varying perturbation because the dynamically assisted Schwinger mechanism is, and accordingly chirality production is expected to be, enhanced more strongly for faster perturbations [74,75]. Indeed, chirality production for a pulsed electric field was discussed in Ref. [110], finding that chirality production (by massive particles) decreases with increasing duration of the electric field. Since no chirality should be produced in the limit of vanishing duration, we can naturally expect that there exists an optimal duration or frequency for the dynamical assistance to maximize chirality production.

The purpose of the present paper is to discuss effects of a parallel slow strong magnetic field on the dynamically assisted Schwinger mechanism and its impacts on chirality production. This shall be achieved in the following manner: In Sec. II, we discuss the number of particles produced from the vacuum by a slow strong parallel electromagnetic field superimposed by a fast weak perturbative electromagnetic field. We derive an analytical formula for the production number by extending the perturbation theory in the Furry picture for a purely slow strong electric field configuration [74,75,84-89] to include a parallel magnetic component. With this formula, we analytically discuss the interplay between the Schwinger mechanism and one-photon pair production and the dynamical assistance between the two production mechanisms in the presence of a parallel slow strong magnetic field. Advantages of the perturbation theory in the Furry picture are that (i) the production number formula is applicable to perturbations with arbitrary time dependence and hence it is valid even for a fast perturbation (the validity was explicitly tested by comparing with a numerical approach in Refs. [74,75,87,88]), which is not accessible with approaches based on adiabatic approximations such as the worldline instanton formalism [79-81]; (ii) one can manifestly discuss the interplay and dynamical assistance between the Schwinger mechanism and one-photon pair production, which is not feasible within other approaches; and (iii) the formula can easily be applied to chirality production. In Sec. III, we discuss chirality production for the same field configuration. We first derive an analytical formula for chirality production by explicitly evaluating an in-in vacuum expectation value of the chirality operator and using the pro- duction number formula. Based on the formula, we investigate how the dynamically assisted Schwinger mechanism affects chirality production. In Sec. IV, we summarize our findings and discuss future work and implications for or applications to, e.g., heavy-ion collisions and intense laser experiments.

\section{PRODUCTION NUMBER}

In this section, we discuss the number of particles produced from the vacuum in the presence of a slow strong electromagnetic field superimposed by a fast weak perturbative electromagnetic field. Our formulation is based on the perturbation theory in the Furry picture [84-86], which was recently applied to a case where a slow strong electromagnetic field is purely electric [74,75,87-89]. We generalize the preceding calculations by including a parallel magnetic component and derive an analytical formula for the production number (see Sec. II A). Based on the formula, we analytically discuss the particle production and clarify how the existence of a slow strong parallel magnetic field affects the interplay between the Schwinger mechanism and one-photon pair production and the dynamical assistance between the two mechanisms (see Secs. II B and II C).

\section{A. Perturbation theory in the Furry picture}

We explain the perturbation theory in the Furry picture by following Ref. [74] and derive a formula for the number of particles produced from the vacuum in the presence of a slow strong electromagnetic field $\bar{A}_{\mu}$ superimposed by a fast weak perturbative electromagnetic field $\mathcal{A}_{\mu} \ll \bar{A}_{\mu}$. In this work, for the sake of simplicity, we neglect back-reaction from produced particles and treat the electromagnetic fields just classically, i.e., higher order quantum processes such as bremsstrahlung are neglected.

We first solve the Dirac equation,

$$
[i \not \partial-e \bar{A}-m] \hat{\psi}=e \mathcal{A} \hat{\psi},
$$

perturbatively in terms of the perturbative field $\mathcal{A}_{\mu}$ while the interaction with the strong field $\bar{A}_{\mu}$ is treated nonperturbatively. To do this, we introduce a (retarded) Green function $S_{\mathrm{R}}$ which is fully dressed by the strong field $\bar{A}_{\mu}$ as

$$
\begin{aligned}
{\left[i \partial_{x}-e \bar{A}(x)-m\right] S_{\mathrm{R}}(x, y) } & =\delta^{4}(x-y), \\
S_{\mathrm{R}}(x, y) & =0 \text { for } x^{0}-y^{0}<0 .
\end{aligned}
$$

With the Green function $S_{\mathrm{R}}$, we can write down a formal solution of the Dirac equation (3) as

$$
\hat{\psi}(x)=\sqrt{Z} \hat{\psi}^{\text {in }}(x)+e \int d^{4} y S_{\mathrm{R}}(x, y) \mathcal{A}(y) \hat{\psi}(y) .
$$

Here, we assumed that the perturbative field $\mathcal{A}_{\mu}$ adiabatically goes off at the infinite past and future and required the Lehmann-Symanzik-Zimmermann (LSZ) asymptotic condition [111]

$$
\lim _{x^{0} \rightarrow-\infty /+\infty} \hat{\psi}(x)=\sqrt{Z} \hat{\psi}^{\text {(in/out) }}
$$

with $Z$ being the field renormalization constant and $\hat{\psi}^{\text {(in/out) }}$ being the asymptotic field operator at $x^{0} \rightarrow-\infty /+\infty$. Notice that $\hat{\psi}^{\text {(in) }} \neq \hat{\psi}^{\text {(out) }}$ in general because of the interaction 
with the electromagnetic fields $\bar{A}_{\mu}$ and $\mathcal{A}_{\mu}$. The asymptotic field operator $\hat{\psi}^{\text {as }}$ (as $=$ in, out) is the solution of the Dirac equation without the perturbative field $\mathcal{A}_{\mu}$ and can be expressed as a mode integral

$$
\hat{\psi}^{(\mathrm{as})}(x)=\bigvee_{i}\left[+\psi_{i}^{(\mathrm{as})}(x) \hat{a}_{i}^{(\mathrm{as})}+{ }_{-} \psi_{i}^{(\mathrm{as})}(x) \hat{b}_{i}^{(\mathrm{as}) \dagger}\right]
$$

where $\mathbb{Z}_{i}$ denotes summation/integration over all the quantum numbers $i$ (e.g., momentum, spin) and the mode functions ${ }_{+} \psi_{i}^{(\text {as) }}$ and ${ }_{-} \psi_{i}^{(\text {as })}$ are the two independent solutions of

$$
0=[i \nexists-e \bar{A}-m]_{ \pm} \psi_{i}^{(\text {as })}
$$

with normalization

$$
\begin{aligned}
\delta_{i, i^{\prime}} & =\int d^{3} \boldsymbol{x}_{ \pm} \psi_{i}^{\left(\text {as }{ }^{\dagger}\right.}{ }_{ \pm} \psi_{i^{\prime}}^{(\text {as })}, \\
0 & =\int d^{3} \boldsymbol{x}_{\mp} \psi_{i}^{\left(\text {as) }{ }^{\dagger}\right.}{ }_{ \pm} \psi_{i^{\prime}}^{(\text {as }} .
\end{aligned}
$$

The subscript \pm specifies the positive and negative frequency modes of the mode function ${ }_{ \pm} \psi_{i}^{(\text {as) }}$. Namely, we identify the positive and negative frequency mode functions at the asymptotic time, ${ }_{+} \psi_{i}^{\text {(as) }}$ and ${ }_{-} \psi_{i}^{\text {(as) }}$, respectively, by

$$
\lim _{x^{0} \rightarrow-\infty /+\infty}{ }^{ \pm} \psi_{i}^{(\mathrm{in} / \mathrm{out}) \dagger} \propto \exp \left[\mp i \int^{x^{0}} d x^{\prime 0} \omega_{i}\right],
$$

where $\omega_{i}>0$ is the one-particle energy at the asymptotic time.

After the canonical quantization procedure, we can interpret $\hat{a}_{i}^{\text {(as) }}$ and $\hat{b}_{i}^{\text {(as) }}$ as annihilation operators of a particle and an antiparticle at the corresponding asymptotic states, respectively. For the normalization (9), the commutation relations for $\hat{a}_{i}^{\text {(as) }}$ and $\hat{b}_{i}^{\text {(as) }}$ read

$$
\begin{aligned}
\delta_{i, i^{\prime}} & =\left\{\hat{a}_{i}^{(\text {as })}, \hat{a}_{i^{\prime}}^{(\text {as }) \dagger}\right\}=\left\{\hat{b}_{i}^{(\text {as })}, \hat{b}_{i^{\prime}}^{(\text {as }) \dagger}\right\}, \\
0 & =\text { (others). }
\end{aligned}
$$

The annihilation operators at the infinite past and future do not coincide with each other in the presence of the external electromagnetic fields $\bar{A}_{\mu}$ and $\mathcal{A}_{\mu}$. Indeed, from the formal solution (5), we find

$$
\begin{aligned}
& \left(\begin{array}{c}
\hat{a}_{i}^{\text {(out) }} \\
\hat{b}_{i}^{\text {(out }) \dagger}
\end{array}\right)=\int d^{3} \boldsymbol{x}\left(\begin{array}{c}
+\psi_{i}^{\text {(out }) \dagger} \\
-\psi_{i}^{\text {(out }) \dagger}
\end{array}\right) \hat{\psi}^{\text {out }} \\
& =\lim _{x^{0} \rightarrow \infty} \int d^{3} \boldsymbol{x}\left(\begin{array}{c}
+\psi_{i}^{(\text {out }) \dagger} \\
-\psi_{i}^{(\text {out })} \dagger
\end{array}\right) \\
& \times\left[\hat{\psi}^{\mathrm{in}}+e \int d^{4} y S_{\mathrm{R}}(x, y) \mathcal{A}(y) \hat{\psi}^{(\mathrm{in})}(y)+\mathcal{O}\left(e^{2}\right)\right] \\
& \equiv \sum_{k=0}^{\infty} e^{k}\left(\begin{array}{c}
\hat{a}_{i}^{(\text {out } ; k)} \\
\hat{b}_{i}^{\text {(out } ; k)} \dagger
\end{array}\right) \text {. }
\end{aligned}
$$

By using

$$
\begin{aligned}
S_{\mathrm{R}}(x, y)= & -i \theta\left(x^{0}-y^{0}\right)\left\{\hat{\psi}^{(\mathrm{as})}(x), \hat{\bar{\psi}}^{(\mathrm{as})}(y)\right\} \\
= & -i \theta\left(x^{0}-y^{0}\right) \\
& \times \oint_{i}\left[{ }_{+} \psi_{i}^{(\mathrm{as})}(x)_{+} \bar{\psi}_{i}^{(\mathrm{as})}(y)+{ }_{-} \psi_{i}^{(\mathrm{as})}(x){ }_{-} \bar{\psi}_{i}^{(\mathrm{as})}(y)\right],
\end{aligned}
$$

we can explicitly write down $\hat{a}_{i}^{(\text {out } ; k)}$ and $\hat{b}_{i}^{(\text {out } ; k)}$. Up to $k \leqslant 1$, we find

$$
\begin{aligned}
& \hat{a}_{i}^{\text {(out; } 0)}=\sum_{i^{\prime}}\left[\left(\int d^{3} \boldsymbol{x}_{+} \psi_{i}^{(\text {out }) \dagger}+\psi_{i^{\prime}}^{(\text {in })}\right) \hat{a}_{i^{\prime}}^{(\text {in })}\right. \\
& \left.+\left(\int d^{3} \boldsymbol{x}_{+} \psi_{i}^{(\mathrm{out}) \dagger}-\psi_{i^{\prime}}^{(\mathrm{in})}\right) \hat{b}_{i^{\prime}}^{(\mathrm{in}) \dagger}\right], \\
& \hat{a}_{i}^{(\text {out } ; 1)}=\sum_{i^{\prime}}\left[\left(-i \int d^{4} x_{+} \bar{\psi}_{i}^{(\text {out })} \mathcal{A}_{+} \psi_{i^{\prime}}^{(\text {in })}\right) \hat{a}_{i^{\prime}}^{(\text {in })}\right. \\
& \left.+\left(-i \int d^{4} x_{+} \bar{\psi}_{i}^{(\text {out })} \mathcal{A}_{-} \psi_{i^{\prime}}^{(\text {in })}\right) \hat{b}_{i^{\prime}}^{(\text {in }) \dagger}\right],
\end{aligned}
$$

and

$$
\begin{aligned}
& \hat{b}_{i}^{(\text {out } ; 0) \dagger}=\sum_{i^{\prime}}\left[\left(\int d^{3} \boldsymbol{x}_{-} \psi_{i}^{(\text {out }) \dagger}+\psi_{i^{\prime}}^{(\text {in })}\right) \hat{a}_{i^{\prime}}^{(\text {in })}\right. \\
& \left.+\left(\int d^{3} \boldsymbol{x}_{-} \psi_{i}^{(\mathrm{out}) \dagger}-\psi_{i^{\prime}}^{(\mathrm{in})}\right) \hat{b}_{i^{\prime}}^{(\mathrm{in}) \dagger}\right], \\
& \hat{b}_{i}^{(\text {out } ; 1) \dagger}=\mathcal{Y}_{i^{\prime}}\left[\left(-i \int d^{4} x_{-} \bar{\psi}_{i}^{(\text {out })} \mathcal{A}_{+} \psi_{i^{\prime}}^{(\text {in })}\right) \hat{a}_{i^{\prime}}^{(\text {in })}\right. \\
& \left.+\left(-i \int d^{4} x_{-} \bar{\psi}_{i}^{(\text {out })} \mathcal{A}_{-} \psi_{i^{\prime}}^{(\text {in })}\right) \hat{b}_{i^{\prime}}^{\text {(in) } \dagger}\right] .
\end{aligned}
$$

Therefore, the annihilation operator at the infinite future, $\hat{a}_{i}^{\text {(out) }}$ or $\hat{b}_{i}^{\text {(out) }}$, becomes a mixture of those at the infinite past, $\hat{a}_{i}^{(\text {in) }}$ and $\hat{b}_{i}^{(\text {in })}$. Notice that the annihilation operators at the infinite future, $\hat{a}_{i}^{\text {(out) }}$ and $\hat{b}_{i}^{\text {(out) }}$, contain the creation operators at the infinite past, $\hat{a}_{i}^{(\mathrm{in}) \dagger}$ and $\hat{b}_{i}^{(\text {out }) \dagger}$, respectively. This means that $\hat{a}_{i}^{\text {(out) }}$ and $\hat{b}_{i}^{\text {(out) }}$ do not annihilate the vacuum state at the infinite past, i.e., the in-vacuum expectation value of the number operator at the infinite future becomes nonvanishing.

We compute the (anti)particle number $N(\bar{N})$ at the infinite future produced from the in-vacuum. Using Eqs. (14) and (15), we can evaluate $N$ and $\bar{N}$ including the first-order nontrivial correction by the perturbative field $\mathcal{A}_{\mu}$ as

$$
\stackrel{(-)}{N}=\bigvee_{i} \stackrel{(-)}{n}_{i},
$$

where $n_{i}$ and $\bar{n}_{i}$ are the distributions of particles and antiparticles per mode $i$, respectively, and are given by

$$
\begin{aligned}
n_{i} & \equiv \frac{\left\langle\text { vac } ; \text { in }\left|\hat{a}_{i}^{(\text {out }) \dagger} \hat{a}_{i}^{(\text {out })}\right| \mathrm{vac} ; \text { in }\right\rangle}{\langle\text { vac; in }| \text { vac } ; \text { in }\rangle} \\
& =\bigvee_{i^{\prime}} \mid\left(\int d^{3} \boldsymbol{x}_{+} \psi_{i}^{\text {(out }) \dagger}-\psi_{i^{\prime}}^{(\text {in })}\right)
\end{aligned}
$$




$$
\begin{aligned}
& -\left.i e\left(\int d^{4} x_{+} \bar{\psi}_{i}^{\text {(out) }} \mathcal{A}_{-} \psi_{i^{\prime}}^{(\text {in })}\right)\right|^{2}, \\
\bar{n}_{i} \equiv & \frac{\left\langle\text { vac; } \text { in }\left|\hat{b}_{i}^{(\text {out })} \hat{b}_{i}^{(\text {out })}\right| \mathrm{vac} ; \text { in }\right\rangle}{\langle\mathrm{vac} ; \text { in }| \mathrm{vac} ; \text { in }\rangle} \\
= & \sum_{i^{\prime}} \mid\left(\int d^{3} x_{-} \psi_{i}^{\text {(out) } \dagger}+\psi_{i^{\prime}}^{\text {(in) }}\right) \\
& -\left.i e\left(\int d^{4} x_{-} \bar{\psi}_{i}^{(\text {out })} \mathcal{A}_{+} \psi_{i^{\prime}}^{(\text {in })}\right)\right|^{2},
\end{aligned}
$$

with $\mid v a c ;$ in $\rangle$ being the vacuum at the infinite past defined as a state such that

$$
\left.\left.0=\hat{a}_{i}^{(\text {in) }} \mid \mathrm{vac} ; \text { in }\right\rangle=\hat{b}_{i}^{(\mathrm{in})} \mid \mathrm{vac} ; \text { in }\right\rangle \quad \text { for any } i .
$$

The first term in Eq. (17) accounts for the Schwinger mechanism because it is independent of the perturbation $\mathcal{A}_{\mu}$ and is driven only by the slow strong electromagnetic field $\bar{A}_{\mu}$. The second term in Eq. (17) accounts for one-photon pair production by the perturbation $\mathcal{A}_{\mu}$. Note that the second term is distinct from the ordinary one-photon pair production since ours is nonperturbatively dressed by the slow strong electromagnetic field $\bar{A}_{\mu}$. As shown below, this dressing enables us to describe the smooth interplay between the Schwinger mechanism and one-photon pair production as well as the dynamical assistance between the two mechanisms.

\section{B. Analytical discussion for a parallel strong electromagnetic field with a perturbation}

To get a qualitative understanding of the particle production, let us consider a spatially homogeneous system and a constant parallel strong electromagnetic field $\overline{\boldsymbol{E}} \| \overline{\boldsymbol{B}}$ superimposed by a fast weak perturbation $\mathcal{E}$ pointing in the same direction. Namely, we consider an electromagnetic field configuration,

$$
\boldsymbol{E}=\left(0,0, \bar{E}+\mathcal{E}\left(x^{0}\right)\right), \quad \boldsymbol{B}=(0,0, \bar{B}),
$$

which is realized by a gauge potential $A_{\mu}=\bar{A}_{\mu}+\mathcal{A}_{\mu}$ such that

$$
\begin{aligned}
\bar{A}^{\mu} & =\left(0,-\bar{B} x^{2}, 0,-\bar{E} x^{0}\right), \\
\mathcal{A}^{\mu} & =\left(0,0,0,-\int^{x^{0}} d x^{\prime 0} \mathcal{E}\left(x^{\prime 0}\right)\right),
\end{aligned}
$$

For simplicity, we assume $e \bar{E}, e \bar{B}>0$. Note that the time dependence of $\mathcal{E}$ is arbitrary at this stage.

\section{Evaluation of the production number formula (16)}

We analytically evaluate the production number formula (16) for the field configuration (20).

First, we solve the Dirac equation (8) in the presence of the parallel strong electromagnetic field $\bar{A}_{\mu}$ (20). This is analytically doable. We find that there are four good quantum numbers $i=p_{1}, p_{3}, n$, and $s$ to label the mode function ${ }_{ \pm} \psi_{i}^{(\text {as) }}={ }_{ \pm} \psi_{p_{1}, p_{3}, n, s}^{(\text {(as) }}$; namely, the canonical momenta $p_{1}$ and $p_{3}$ (i.e., the eigenvalues of the translation operators $-i \partial_{1}$ and $-i \partial_{3}$, respectively), the Landau level $n=0,1,2, \ldots \in \mathbb{N}$ (i.e., the remaining transverse momentum $p_{2}$ is quantized by the magnetic field), and spin $s= \pm 1$. We can write down the mode function ${ }_{ \pm} \psi_{p_{1}, p_{3}, n, s}^{(\text {as })}$ explicitly as

$$
\begin{aligned}
\left(\begin{array}{c}
+\psi_{p_{1}, p_{3}, n, s}^{(\mathrm{as})}(x) \\
-\psi_{p_{1}, p_{3}, n, s}^{(\mathrm{as})}(x)
\end{array}\right)= & \left(\begin{array}{cc}
A_{p_{1}, p_{3}, n, s}^{(\mathrm{as})}\left(x^{0}\right) & B_{p_{1}, p_{3}, n, s}^{(\mathrm{as})}\left(x^{0}\right) \\
B_{p_{1}, p_{3}, n, s}^{(\mathrm{as}) *}\left(x^{0}\right) & -A_{p_{1}, p_{3}, n, s}^{\text {(as) }}\left(x^{0}\right)
\end{array}\right) \\
& \times\left(\begin{array}{l}
U_{p_{1}, n, s}\left(x^{2}\right) \\
V_{p_{1}, n, s}\left(x^{2}\right)
\end{array}\right) \frac{e^{i p_{1} x^{1}} e^{i p_{3} x^{3}}}{2 \pi}
\end{aligned}
$$

Here, the scalar functions $A_{p_{1}, p_{3}, n, s}^{(\mathrm{as})}$ and $B_{p_{1}, p_{3}, n, s}^{(\mathrm{as})}$ are given by

$$
\begin{aligned}
A_{p_{1}, p_{3}, n, s}^{(\text {in })}\left(x^{0}\right) \equiv & e^{-\frac{i \pi}{8}} e^{-\frac{\pi}{8} \frac{m_{\perp}^{2}}{e \bar{E}}} \frac{m_{\perp}}{\sqrt{2 e \bar{E}}} \\
& \times D_{\frac{i}{2} \frac{m_{\perp}^{2}}{e \bar{E}}-1}\left(-e^{-\frac{i \pi}{4}} \sqrt{\frac{2}{e \bar{E}}}\left(p_{3}+e \bar{E} x^{0}\right)\right), \\
B_{p_{1}, p_{3}, n, s}^{\text {(in })}\left(x^{0}\right) \equiv & e^{+\frac{i \pi}{8}} e^{-\frac{\pi}{8} \frac{m_{\perp}^{2}}{e \bar{E}}} \\
& \times D_{\frac{i}{2} \frac{m_{\perp}^{2}}{e \bar{E}}}\left(-e^{-\frac{i \pi}{4}} \sqrt{\frac{2}{e \bar{E}}}\left(p_{3}+e \bar{E} x^{0}\right)\right), \\
A_{p_{1}, p_{3}, n, s}^{\text {(out) }}\left(x^{0}\right) \equiv & e^{-\frac{i \pi}{8}} e^{-\frac{\pi}{8} \frac{m_{\perp}^{2}}{e \bar{E}}} \\
& \times D_{-\frac{i}{2} \frac{m_{\perp}^{2}}{e \bar{E}}}\left(e^{\frac{i \pi}{4}} \sqrt{\frac{2}{e \bar{E}}}\left(p_{3}+e \bar{E} x^{0}\right)\right), \\
B_{p_{1}, p_{3}, n, s}^{\text {(out })}\left(x^{0}\right) \equiv & e^{+\frac{i \pi}{8}} e^{-\frac{\pi}{8} \frac{m_{1}^{2}}{e \bar{E}}} \frac{m_{\perp}}{\sqrt{2 e \bar{E}}} \\
& \times D_{-\frac{i}{2} \frac{m_{1}^{2}}{e \bar{E}}-1}\left(e^{\frac{i \pi}{4}} \sqrt{\frac{2}{e \bar{E}}}\left(p_{3}+e \bar{E} x^{0}\right)\right),
\end{aligned}
$$

with $D_{v}(z)$ being the parabolic cylinder function and $m_{\perp}$ being the transverse mass,

$$
m_{\perp} \equiv \sqrt{m^{2}+e \bar{B}(2 n+1-s)} .
$$

The spinors $U_{p_{1}, n, s}, V_{p_{1}, n, s}$ are given by

$$
\begin{aligned}
U_{p_{1}, n, s}\left(x^{2}\right) \equiv & u_{p_{1}, n}\left(x^{2}\right) \times \Gamma_{s}, \\
V_{p_{1}, n, s}\left(x^{2}\right) \equiv & {\left[\gamma^{0} \frac{m}{m_{\perp}} u_{p_{1}, n}\left(x^{2}\right)\right.} \\
& \left.+\gamma^{1} \frac{\sqrt{e \bar{B}(2 n+1-s)}}{m_{\perp}} u_{p_{1}, n-s}\left(x^{2}\right)\right] \times \Gamma_{s},
\end{aligned}
$$

where

$$
u_{p_{1}, n}\left(x^{2}\right) \equiv \sqrt{\frac{L}{2 \pi}}\left(\frac{e \bar{B}}{\pi}\right)^{\frac{1}{4}} \frac{1}{\sqrt{n !}} D_{n}\left(\sqrt{\frac{2}{e \bar{B}}}\left(p_{1}+e \bar{B} x^{2}\right)\right)
$$

with $L$ being the system size in the $x^{2}$ direction and $\Gamma_{s}$ being an eigenvector of $\gamma^{0} \gamma^{3}$ and $\gamma^{1} \gamma^{2}$ satisfying

$$
\gamma^{0} \gamma^{3} \Gamma_{s}=+\Gamma_{s}, \gamma^{1} \gamma^{2} \Gamma_{s}=-i s \Gamma_{s}, \delta_{s, s^{\prime}}=\Gamma_{s}^{\dagger} \Gamma_{s^{\prime}} .
$$

Note that we normalized $A_{p_{1}, p_{3}, n, s}, B_{p_{1}, p_{3}, n, s}$, and $u_{p_{1}, n}$ as

$$
\begin{aligned}
1 & =\left|A_{p_{1}, p_{3}, n, s}^{\text {(as) }}\right|^{2}+\left|B_{p_{1}, p_{3}, n, s}^{(\mathrm{as})}\right|^{2}, \\
\frac{L}{2 \pi} \delta_{n, n^{\prime}} & =\int d y u_{p_{1}, n^{\prime}}^{\dagger} u_{p_{1}, n},
\end{aligned}
$$


so that the normalization condition (9) for the mode function ${ }_{ \pm} \psi_{p_{1}, p_{3}, n, s}^{(\text {as) }}$ is satisfied as

$$
\delta_{i, i^{\prime}}=\delta_{s, s^{\prime}} \times \delta\left(p_{1}-p_{1}^{\prime}\right) \delta\left(p_{3}-p_{3}^{\prime}\right) \times \frac{L}{2 \pi} \delta_{n, n^{\prime}} .
$$

Note that $(L / 2 \pi) \delta_{n, n^{\prime}}$ corresponds to $\delta\left(p_{2}-p_{2}^{\prime}\right)$ in systems without any magnetic field. Also, note that $A_{p_{1}, p_{3}, n, s}^{(\text {in }} \neq$ $A_{p_{1}, p_{3}, n, s}^{\text {(out) }}$ and $B_{p_{1}, p_{3}, n, s}^{\text {(in) }} \neq B_{p_{1}, p_{3}, n, s}^{\text {(out) }}$ because of the existence of the strong electric field. This is essentially because the Dirac equation becomes time dependent if $e \bar{E} \neq 0$ and then the eigenfunction of the time-translation operator at the infinite past cannot be the one at the infinite future. Physically, this means that the strong electric field supplies energy to the system, which mixes up particle and antiparticle modes.

In terms of the four good quantum numbers $i=p_{1}, p_{3}, n$, and $s$, we can express the mode integral (7) as

$$
\begin{aligned}
\hat{\psi}= & \sum_{s= \pm 1} \frac{2 \pi}{L} \sum_{n=0}^{\infty} \int d p_{1} d p_{3} \\
& \times\left[{ }_{+} \psi_{p_{1}, p_{3}, n, s}^{\text {(as) }} \hat{a}_{p_{1}, p_{3}, n, s}^{(\mathrm{as})}+{ }_{-} \psi_{p_{1}, p_{3}, n, s}^{(\mathrm{as})} \hat{b}_{-p_{1},-p_{3}, n,-s}^{(\mathrm{as}) \dagger}\right] .
\end{aligned}
$$

We assigned the minus signs in the labels of $\hat{b}_{-p_{1},-p_{3}, n,-s}^{\text {(as) }}$. Those minus signs are necessary so that the creation operator $\hat{b}_{p_{1}, p_{3}, n, s}^{\text {(as) }}$ correctly creates a one-particle state with quantum numbers $p_{1}, p_{3}, n$, and $s$. One can check this statement by explicitly computing an expectation value of the corresponding operator at the asymptotic states $\hat{O}^{\text {(as) }}$ [e.g., the canonical momentum operator $\left.\hat{\psi}^{(\text {as }) \dagger}(-i \partial) \hat{\psi}^{(\text {as })}\right]$ with respect to the one-particle state $\hat{b}_{p_{1}, p_{3}, n, s}^{(a s) \dagger} \mid$ vac; as $\rangle$ [17]. According to this assignment, we also have minus signs in the labels of $\bar{n}(17 \mathrm{~b})$ as

$$
\begin{aligned}
& \bar{n}_{-p_{1},-p_{3}, n,-s}=\frac{\left\langle\mathrm{vac} ; \text { in }\left|\hat{b}_{-p_{1},-p_{3}, n,-s}^{(\mathrm{out}) \dagger} \hat{b}_{-p_{1},-p_{3}, n,-s}^{(\mathrm{out})}\right| \mathrm{vac} ; \text { in }\right\rangle}{\langle\mathrm{vac} ; \text { in }| \mathrm{vac} ; \text { in }\rangle} \\
& =\sum_{s^{\prime}= \pm 1} \frac{2 \pi}{L} \sum_{n^{\prime}=0}^{\infty} \int d p_{1}^{\prime} d p_{3}^{\prime} \\
& \times \mid\left(\int d^{3} \boldsymbol{x}_{-} \psi_{p_{1}, p_{3}, n, s+}^{(\text {out }) \dagger} \psi_{p_{1}^{\prime}, p_{3}^{\prime}, n^{\prime}, s^{\prime}}^{(\text {in }}\right) \\
& -\left.i e\left(\int d^{4} x_{-} \bar{\psi}_{p_{1}, p_{3}, n, s}^{\text {(out) }} \mathcal{A}_{+} \psi_{p_{1}^{\prime}, p_{3}^{\prime}, n^{\prime}, s^{\prime}}^{\text {(in) }}\right)\right|^{2} \text {. }
\end{aligned}
$$

Second, we evaluate the production number formula (16) using the analytical expression for the mode function ${ }_{ \pm} \psi_{p_{1}, p_{3}, n, s}^{\text {(as) }}$ (29). By substituting Eq. (29) into Eq. (17), one can show

$$
\begin{aligned}
n_{p_{1}, p_{3}, n, s}= & \bar{n}_{-p_{1},-p_{3}, n,-s}=\frac{V}{(2 \pi)^{3}} \exp \left[-\pi \frac{m_{\perp}^{2}}{e \bar{E}}\right] \\
& \times \mid 1+\frac{1}{2} \frac{m_{\perp}^{2}}{e \bar{E}} \int_{0}^{\infty} d \omega \frac{\tilde{\mathcal{E}}(\omega)}{\bar{E}} \exp \left[-\frac{i}{4} \frac{\omega^{2}+4 \omega p_{3}}{e \bar{E}}\right] \\
& \times\left.{ }_{1} \tilde{F}_{1}\left(1-\frac{i}{2} \frac{m_{\perp}^{2}}{e \bar{E}} ; 2 ; \frac{i}{2} \frac{\omega^{2}}{e \bar{E}}\right)\right|^{2},
\end{aligned}
$$

where $V$ is the space volume of the system,

$$
\tilde{\mathcal{E}}(\omega) \equiv \int d x^{0} e^{-i \omega x^{0}} \mathcal{E}\left(x^{0}\right)
$$

is the Fourier component of the perturbation $\mathcal{E}$, and ${ }_{1} \tilde{F}_{1}(a ; b ; z) \equiv{ }_{1} F_{1}(a ; b ; z) / \Gamma(b)$ is the regularized hypergeometric function. Note that $n_{p_{1}, p_{3}, n, s}=\bar{n}_{-p_{1},-p_{3}, n,-s}$ holds. This is physically because a particle and an antiparticle are always produced as a pair from the vacuum and hence the total amounts of the charge, momentum, and spin of the pair should be vanishing. By integrating Eq. (31) over the quantum numbers, we arrive at

$$
\begin{aligned}
\stackrel{(-)}{N}= & \sum_{s= \pm 1} \frac{2 \pi}{L} \sum_{n=0}^{\infty} \int d p_{1} d p_{3} \stackrel{(-)}{n}_{p_{1}, p_{3}, n, s} \\
= & m^{4} V T \times \frac{1}{4 \pi^{2}} \frac{e \bar{E}}{m^{2}} \frac{e \bar{B}}{m^{2}} \sum_{s= \pm 1} \sum_{n=0}^{\infty} \exp \left[-\pi \frac{m_{\perp}^{2}}{e \bar{E}}\right] \\
& \times\left[1+\frac{2 \pi}{T}\left\{\frac{1}{2} \frac{m_{\perp}^{2}}{e \bar{E}} \frac{\tilde{\mathcal{E}}(0)}{\bar{E}}+\frac{1}{4}\left(\frac{m_{\perp}^{2}}{e \bar{E}}\right)^{2} \int_{0}^{\infty} d \omega\right.\right. \\
& \left.\left.\times\left|\frac{\tilde{\mathcal{E}}(\omega)}{\bar{E}}{ }_{1} \tilde{F}_{1}\left(1-\frac{i}{2} \frac{m_{\perp}^{2}}{e \bar{E}} ; 2 ; \frac{i}{2} \frac{\omega^{2}}{e \bar{E}}\right)\right|^{2}\right\}\right],
\end{aligned}
$$

where $T$ is the whole time interval, and we used $\int d p_{1}=e \bar{B} L$, which accounts for the degeneracy of the Landau level. Notice

that the total production number $\stackrel{(-)}{N}$ depends on the magnetic field $e \bar{B}$ through (i) the overall factor $e \bar{B}$, which comes from the phase space under the Landau quantization $\int d^{2} \boldsymbol{p}_{\perp} \rightarrow$ $(e \bar{B} / 2 \pi) \sum_{n}$; and (ii) the transverse mass $m_{\perp}$, which is discretized by the Landau quantization as $\sqrt{m^{2}+\boldsymbol{p}_{\perp}^{2}} \rightarrow$ $\sqrt{m^{2}+e \bar{B}(2 n+1-s)}=m_{\perp}$. This means that the effect of the parallel strong magnetic field $e \bar{B}$ is just to discretize the transverse momentum via the Landau quantization, and one may say that the particle production (or the dynamically assisted Schwinger mechanism) is essentially unaffected by the presence of a strong magnetic field. This is a reasonable result since magnetic fields cannot do work on a charged particle (i.e., cannot supply energy to vacuum fluctuations to produce real particles), and they are just able to change motion of the particle (i.e., can change momentum). Nevertheless, the change of momentum can result in nontrivial physics consequences such as chirality production, which we discuss in Sec. III.

Below, let us consider some limiting situations to analytically understand basic features of the production number formula (34). In particular, we clarify the relationship between the formula (34) and the well-established production formulas for the Schwinger mechanism and one-photon pair production in terms of the size of the physical parameters such as the electromagnetic field strength and the typical slowness/fastness of a perturbation.

\section{Dependence on the electric field $\bar{e} \bar{E}$}

The electric field strength $e \bar{E}$ controls the interplay between the Schwinger mechanism and one-photon pair production.

When the strong electric field becomes very strong, $e \bar{E} \rightarrow$ $\infty$, the Schwinger mechanism becomes free from the exponential suppression by mass and can produce particles abundantly. As a result, the particle production is dominated 
by the Schwinger mechanism, i.e., the first term in Eq. (31) dominates the production as

$$
n_{p_{1}, p_{3}, n, s}=\bar{n}_{-p_{1},-p_{3}, n,-s} \stackrel{e \bar{E} \rightarrow \infty}{\longrightarrow} \frac{V}{(2 \pi)^{3}} \exp \left[-\pi \frac{m_{\perp}^{2}}{e \bar{E}}\right],
$$

which yields

$$
\begin{aligned}
N=\bar{N} \stackrel{e \bar{E} \rightarrow \infty}{\longrightarrow} & m^{4} V T \times \frac{1}{4 \pi^{2}} \frac{e \bar{B}}{m^{2}} \frac{e \bar{E}}{m^{2}} \\
& \times \exp \left[-\pi \frac{m^{2}}{e \bar{E}}\right] \operatorname{coth}\left[\pi \frac{e \bar{B}}{e \bar{E}}\right] \\
\equiv & N_{\text {Schwinger }} .
\end{aligned}
$$

This agrees exactly with the Schwinger formula in the presence of a strong parallel magnetic field (2) [6,11-17].

In case that the strong electric field is not so strong or absent, $e \bar{E} \rightarrow 0$, the Schwinger mechanism does not take place. The particle production is, then, driven solely by onephoton pair production by the perturbation $e \mathcal{E}$ without any modification from the strong electric field $e \bar{E}$ but with the Landau quantization by the strong magnetic field $e \bar{B}$. Namely, only the second term in Eq. (31) contributes to the production, and the distribution $\stackrel{(-)}{n}_{p_{1}, p_{3}, n, s}$ reads

$$
\begin{aligned}
& n_{p_{1}, p_{3}, n, s}=\bar{n}_{-p_{1},-p_{3}, n,-s} \\
& \stackrel{e \bar{E} \rightarrow 0}{\longrightarrow} \frac{V}{(2 \pi)^{3}} \frac{1}{4} \frac{m_{\perp}^{2}}{m_{\perp}^{2}+p_{3}^{2}} \frac{\left|e \tilde{\mathcal{E}}\left(2 \sqrt{m_{\perp}^{2}+p_{3}^{2}}\right)\right|^{2}}{m_{\perp}^{2}+p_{3}^{2}} .
\end{aligned}
$$

By integrating Eq. (37), we find that the total production number $\stackrel{(-)}{N}$ is given by

$$
\begin{aligned}
N= & \bar{N} \stackrel{e \bar{E} \rightarrow 0}{\longrightarrow} m^{3} V \times \frac{1}{16 \pi^{2}} \frac{g \bar{B}}{m^{2}} \sum_{s= \pm 1} \sum_{n=0}^{\infty} \int \frac{d p_{3}}{m} \\
& \times \frac{m_{\perp}^{2}}{m_{\perp}^{2}+p_{3}^{2}} \frac{\left|e \tilde{\mathcal{E}}\left(2 \sqrt{m_{\perp}^{2}+p_{3}^{2}}\right)\right|^{2}}{m_{\perp}^{2}+p_{3}^{2}} .
\end{aligned}
$$

Notice that the argument of $e \tilde{\mathcal{E}}$ is $2 \sqrt{m_{\perp}^{2}+p_{3}^{2}}$, i.e., the perturbative electric field $e \mathcal{E}$ needs to supply energy $2 \times$ $\sqrt{m_{\perp}^{2}+p_{3}^{2}}$ to produce a pair of particles. ${ }^{2}$ This means that one-photon pair production never occurs if the frequency $\Omega$ of the perturbative electric field is below the lowest energy level $\Omega<2 m$, and that the production number increases sharply whenever the frequency matches the $n$th energy level $\Omega=$ $2 m_{\perp}=2 \sqrt{m^{2}+e \bar{B}(2 n+1-s)}$. In the presence of the strong electric field $e \bar{E} \neq 0$, these threshold behaviors are smeared because of the energy supply by $e \bar{E}$, which can be interpreted as the dynamical assistance to one-photon pair production

\footnotetext{
${ }^{2} n$-photon pair production processes $(n>1)$ have lower thresholds $2 \times \sqrt{m_{\perp}^{2}+p_{3}^{2}} / n$. Such higher order processes are parametrically suppressed by $\left(e \mathcal{E} / \mathrm{m}^{2}\right)^{2 n}$, so that they are negligible as long as the perturbation $e \mathcal{E}$ is sufficiently weak, and thus the dominant contribution to the production number always comes from the one-photon pair production $(n=1)$.
}

by the Schwinger mechanism and vice versa, as we shall demonstrate explicitly in Sec. II C.

\section{Dependence on the magnetic field $e \bar{B}$}

The effect of the magnetic field is just to discretize the energy level, and the energy difference among the levels increases with the magnetic field strength $e \bar{B}$. Therefore, the number of the Landau levels that contribute to the particle production changes with $e \bar{B}$. Unlike the electric field strength $e \bar{E}$, the magnetic field strength $e \bar{B}$ cannot control the interplay between the Schwinger mechanism and one-photon pair production because the relative size between the two mechanisms is independent of $e \bar{B}$.

For a very strong magnetic field $e \bar{B} \rightarrow \infty$, the lowest Landau level $n=0, s= \pm 1$ (+ for particle, and - for antiparticle) dominates the production since the contributions from the higher Landau levels are exponentially suppressed by $e \bar{B}$. Therefore, we have

$$
\begin{aligned}
n_{p_{1}, p_{3}, n, s}= & \bar{n}_{-p_{1},-p_{3}, n,-s} \\
\stackrel{e \bar{B} \rightarrow \infty}{\longrightarrow} & \delta_{n, 0} \delta_{s,+1} \times \frac{V}{(2 \pi)^{3}} \exp \left[-\pi \frac{m^{2}}{e \bar{E}}\right] \\
& \times \mid 1+\frac{1}{2} \frac{m^{2}}{e \bar{E}} \int_{0}^{\infty} d \omega \frac{\tilde{\mathcal{E}}(\omega)}{\bar{E}} \exp \left[-\frac{i}{4} \frac{\omega^{2}+4 \omega p_{3}}{e \bar{E}}\right] \\
& \times\left.{ }_{1} \tilde{F}_{1}\left(1-\frac{i}{2} \frac{m^{2}}{e \bar{E}} ; 2 ; \frac{i}{2} \frac{\omega^{2}}{e \bar{E}}\right)\right|^{2},
\end{aligned}
$$

which yields

$$
\begin{aligned}
& \stackrel{(-)}{N} \stackrel{e \bar{B} \rightarrow \infty}{\longrightarrow} \stackrel{(-)}{N}_{\text {LLL }} \\
& \equiv m^{4} V T \times \frac{1}{4 \pi^{2}} \frac{e \bar{E}}{m^{2}} \frac{e \bar{B}}{m^{2}} \exp \left[-\pi \frac{m^{2}}{e \bar{E}}\right] \\
& \times {\left[1+\frac{2 \pi}{T}\left\{\frac{1}{2} \frac{m^{2}}{e \bar{E}} \frac{\tilde{\mathcal{E}}(0)}{\bar{E}}+\frac{1}{4}\left(\frac{m^{2}}{e \bar{E}}\right)^{2} \int_{0}^{\infty} d \omega\right.\right.} \\
&\left.\left.\times\left|\frac{\tilde{\mathcal{E}}(\omega)}{\bar{E}}{ }_{1} \tilde{F}_{1}\left(1-\frac{i}{2} \frac{m^{2}}{e \bar{E}} ; 2 ; \frac{i}{2} \frac{\omega^{2}}{e \bar{E}}\right)\right|^{2}\right\}\right] .
\end{aligned}
$$

The strong magnetic field $e \bar{B}$ appears just as an overall factor in Eq. (40) because of the enhancement of the phase space $\propto e \bar{B}$. Thus, the production number $\stackrel{(-)}{N}$ linearly increases with $e \bar{B}$ if the magnetic field is very strong.

The lowest Landau level production $\stackrel{(-)}{N}_{\text {LLL }}$ is dominated by the Schwinger mechanism [i.e., first term in Eq. (40)] in the chiral limit $m \rightarrow 0$ as

$$
\stackrel{(-)}{N_{\text {LLL }}} \stackrel{m \rightarrow 0}{\longrightarrow} V T \times \frac{e \bar{E} e \bar{B}}{4 \pi^{2}} .
$$

This is because the lowest Landau level is gapless in the chiral limit. Therefore, the phase-space of particles is already occupied by the production from the Schwinger mechanism, and the additional production by the perturbative electric field is forbidden by the Pauli principle.

If the magnetic field is not so strong or absent, $e \bar{B} \rightarrow 0$, the transverse momentum becomes continuous, and hence the summation over the Landau level $n$ may be written as an 
integration over the transverse momentum. Namely, one may replace the summation $e \bar{B} \sum_{n}$ in Eq. (34) with an integration $\int d p_{\perp} p_{\perp}$ with $p_{\perp}^{2} \equiv e \bar{B}(2 n+1-s)$ as

$$
\begin{aligned}
\stackrel{(-)}{N} & \stackrel{e \bar{B} \rightarrow 0}{\longrightarrow} \sum_{s= \pm 1} \int d^{3} \boldsymbol{p} \stackrel{(-)}{n}_{p_{1}, p_{2}, p_{3}, s} \\
= & m^{4} V T \times \frac{1}{2 \pi^{2}} \frac{e \bar{E}}{m^{2}} \int_{0}^{\infty} \frac{d p_{\perp} p_{\perp}}{m^{2}} \exp \left[-\pi \frac{m^{2}+p_{\perp}^{2}}{e \bar{E}}\right] \\
& \times\left[1+\frac{2 \pi}{T}\left\{\frac{1}{2} \frac{m^{2}+p_{\perp}^{2}}{e \bar{E}(0)} \frac{\tilde{E}}{\bar{E}}+\frac{1}{4}\left(\frac{m^{2}+p_{\perp}^{2}}{e \bar{E}}\right)^{2} \int_{0}^{\infty} d \omega\right.\right. \\
& \left.\left.\times\left|\frac{\tilde{\mathcal{E}}(\omega)}{\bar{E}}{ }_{1} \tilde{F}_{1}\left(1-\frac{i m^{2}+p_{\perp}^{2}}{2} ; 2 ; \frac{i}{2} \frac{\omega^{2}}{e \bar{E}}\right)\right|^{2}\right\}\right] .
\end{aligned}
$$

Equation (42) exactly agrees with the result of Ref. [74], in which the production number formula (34) for a purely slow strong electric field was derived.

Before continuing, it is instructive for later discussions to take the $e \bar{E} \rightarrow 0$ limit of Eq. (42), i.e., to derive a formula for one-photon pair production without any strong electromagnetic field. We find

$$
\begin{aligned}
& N=\bar{N} \stackrel{e \bar{E}, e \bar{B} \rightarrow 0}{\longrightarrow} m^{3} V \times \frac{1}{4 \pi^{2}} \int_{0}^{\infty} \frac{d p}{m} \frac{p^{2}}{m^{2}} \\
& \times \frac{m^{2}+\frac{2}{3} p^{2}}{m^{2}+p^{2}} \frac{\left|e \tilde{\mathcal{E}}\left(2 \sqrt{m^{2}+p^{2}}\right)\right|^{2}}{m^{2}+p^{2}} \\
& \equiv N_{\text {one-photon }} \text {, }
\end{aligned}
$$

where $p^{2} \equiv p_{\perp}^{2}+p_{3}^{2}$. In contrast to the one-photon pair production in the presence of $e \bar{B} \neq 0$ [Eq. (38)], the energy level is continuous in Eq. (43). Hence, the production number does not exhibit any threshold behaviors in terms of the frequency of the perturbation. In other words, addition of a strong parallel magnetic field results in sharp peak structures in the production number as a function of the frequency because of the Landau quantization.

\section{Dependence on the frequency of the perturbation $\Omega$}

The typical frequency of a perturbation, which we write as $\Omega$, can control the interplay between the Schwinger mechanism and one-photon pair production. Intuitively, the energy supplied by a perturbation via a one-photon process is $\Omega$. Therefore if the supplied energy by a perturbation $\Omega$ becomes much larger (smaller) than that by the strong electric field $e \bar{E}$, one-photon pair production (the Schwinger mechanism) should dominate the production. The strong magnetic field is not essential for this interplay since it cannot supply energy as discussed in the previous subsections.

Suppose that the frequency is very small, $\Omega \rightarrow 0$. This is equivalent to assuming

$$
\mathcal{E}\left(x^{0}\right)=\mathcal{E}_{0} \Leftrightarrow \tilde{\mathcal{E}}(\omega)=\mathcal{E}_{0} \times 2 \pi \delta(\omega) .
$$

Then, we can analytically carry out the $\omega$ integration in Eq. (31) as

$$
\begin{aligned}
n_{p_{1}, p_{3}, n, s} & =\bar{n}_{-p_{1},-p_{3}, n,-s} \\
& \stackrel{\Omega \rightarrow 0}{\longrightarrow} \frac{V}{(2 \pi)^{3}} \exp \left[-\pi \frac{m_{\perp}^{2}}{e \bar{E}}\right]\left|1+\frac{\pi}{2} \frac{m_{\perp}^{2}}{e \bar{E}} \frac{\mathcal{E}_{0}}{\bar{E}}\right|^{2} \\
& =\frac{V}{(2 \pi)^{3}} \exp \left[-\pi \frac{m_{\perp}^{2}}{e\left(\bar{E}+\mathcal{E}_{0}\right)}\right]^{2}+\mathcal{O}\left(\left(\mathcal{E}_{0} / \bar{E}\right)^{2}\right)
\end{aligned}
$$

Therefore, we can evaluate the total production number $\stackrel{(-)}{N}$ as

$$
\begin{aligned}
N= & \bar{N} \stackrel{\Omega \rightarrow 0}{\longrightarrow} m^{4} V T \times \frac{1}{4 \pi^{2}} \frac{e \bar{B}}{m^{2}} \frac{e\left(\bar{E}+\mathcal{E}_{0}\right)}{m^{2}} \\
& \times \exp \left[-\pi \frac{m^{2}}{e\left(\bar{E}+\mathcal{E}_{0}\right)}\right] \operatorname{coth}\left[\pi \frac{e \bar{B}}{e\left(\bar{E}+\mathcal{E}_{0}\right)}\right],
\end{aligned}
$$

where $\mathcal{O}\left(\left(\mathcal{E}_{0} / \bar{E}\right)^{2}\right)$ correction is neglected. By noting that the total electric field strength for $\Omega \rightarrow 0$ (44) is $E=\bar{E}+\mathcal{E}_{0}$, we see that Eq. (46) agrees with the well-established Schwinger formula (36). Therefore, the particle production is, indeed, dominated by the Schwinger mechanism if the frequency $\Omega$ is small.

If the frequency is very large $\Omega \rightarrow \infty$, the $\omega$ integration in Eq. (31) is dominated by $\omega=\Omega \rightarrow \infty$ modes. Thus, we find

$$
\begin{aligned}
& n_{p_{1}, p_{3}, n, s}= \bar{n}_{-p_{1},-p_{3}, n,-s} \\
& \stackrel{\Omega \rightarrow \infty}{\longrightarrow} \frac{V}{(2 \pi)^{3}} \mid \exp \left[-\frac{\pi}{2} \frac{m_{\perp}^{2}}{e \bar{E}}\right] \\
&+\left.\frac{1}{2} \frac{m_{\perp}}{\sqrt{m_{\perp}^{2}+p_{3}^{2}}} \frac{e \tilde{\mathcal{E}}\left(2 \sqrt{m_{\perp}^{2}+p_{3}^{2}}\right)}{\sqrt{m_{\perp}^{2}+p_{3}^{2}}}\right|^{2} \\
& \stackrel{e \bar{E} \lesssim m_{\perp}^{2}}{\longrightarrow} \frac{V}{(2 \pi)^{3}} \times \frac{1}{4} \frac{m_{\perp}^{2}}{m_{\perp}^{2}+p_{3}^{2}} \frac{\left|e \tilde{\mathcal{E}}\left(2 \sqrt{m_{\perp}^{2}+p_{3}^{2}}\right)\right|^{2}}{m_{\perp}^{2}+p_{3}^{2}},
\end{aligned}
$$

which yields

$$
\begin{aligned}
N= & \bar{N} \\
\stackrel{\Omega \rightarrow \infty, e \bar{E} \lesssim m_{\perp}^{2}}{\longrightarrow} & m^{3} V \times \frac{1}{16 \pi^{2}} \frac{e \bar{B}}{m^{2}} \sum_{s= \pm 1} \sum_{n=0}^{\infty} \int \frac{d p_{3}}{m} \\
& \times \frac{m_{\perp}^{2}}{m_{\perp}^{2}+p_{3}^{2}} \frac{\left|e \tilde{\mathcal{E}}\left(2 \sqrt{m_{\perp}^{2}+p_{3}^{2}}\right)\right|^{2}}{m_{\perp}^{2}+p_{3}^{2}} .
\end{aligned}
$$

To get Eq. (47), we neglected the first term in the first line (i.e., the contribution from the Schwinger mechanism), which is exponentially suppressed by $e \bar{E} / m_{\perp}^{2}$, assuming that $e \bar{E}$ is not so strong $e \bar{E} \lesssim m_{\perp}^{2}$. If this condition is satisfied, Eq. (48) agrees with Eq. (38), i.e., the production is dominated by one-photon pair production. If $e \bar{E}$ is very strong $e \bar{E} \gtrsim m_{\perp}^{2}$, the strong electric field can supply very large energy to the vacuum, so that the Schwinger mechanism always dominates the production no matter how fast a perturbation is, as we discussed in Sec. II B 2. 

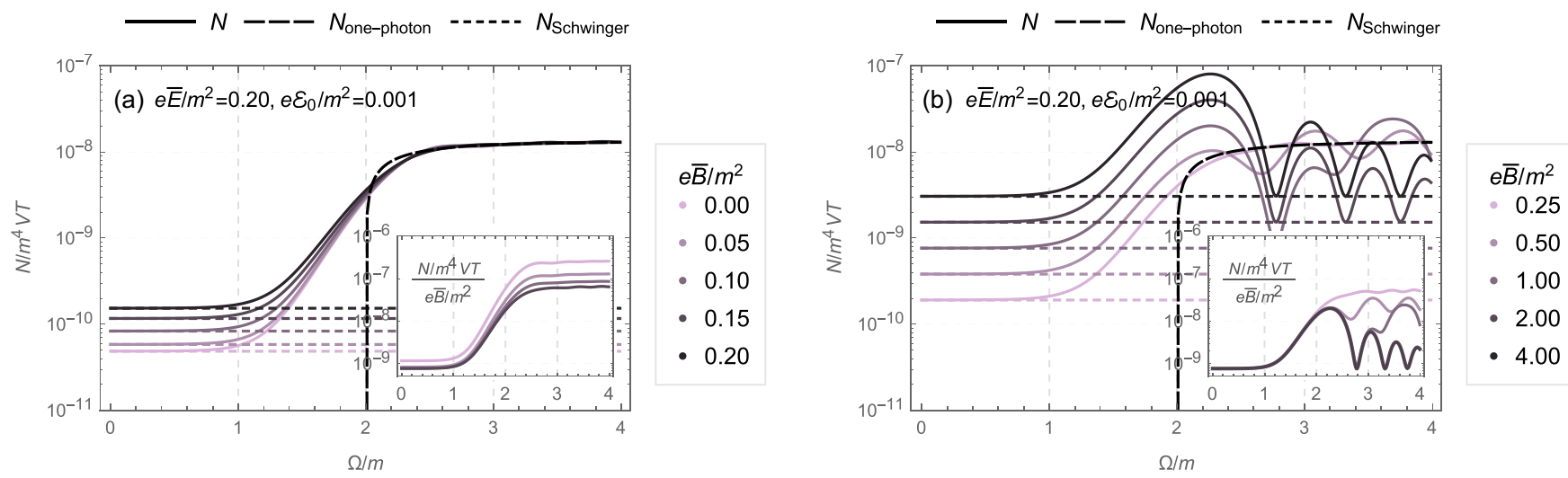

FIG. 2. The total production number $N$ (50) for the monochromatic perturbation (49) as a function of the frequency $\Omega / m$ for various values of the magnetic field strength $e \bar{B} / m^{2}=0.00,0.05,0.10,0.15,0.20$ (a) and $0.25,0.50,1.00,2.00,4.00$ (b). The small panels show the total production number scaled by the magnetic field strength $N /\left(e \bar{B} / \mathrm{m}^{2}\right)$. The other parameters are fixed as $e \bar{E} / \mathrm{m}^{2}=0.20$ and $e \mathcal{E}_{0} / \mathrm{m}^{2}=0.001$. For comparison, the one-photon pair production formula $N_{\text {one-photon }}$ (43) and the Schwinger formula $N_{\text {Schwinger }}(36)$ are plotted by the dashed and dotted lines, respectively.

\section{Quantitative discussion for a parallel strong electromagnetic field with a monochromatic perturbation}

To understand more detailed features of the particle production, let us consider, as a demonstration, the parallel electromagnetic field configuration (20) with a monochromatic perturbation,

$$
\begin{aligned}
\mathcal{E}\left(x^{0}\right) & =\mathcal{E}_{0} \cos \left(\Omega x^{0}+\phi\right) \\
\Leftrightarrow \tilde{\mathcal{E}}(\omega) & =\pi \mathcal{E}_{0} \sum_{ \pm} e^{ \pm i \phi} \delta(\Omega \mp \omega) .
\end{aligned}
$$

For this perturbation, one can analytically carry out the $\omega$ integration in the production number formula (34) as

$$
\begin{aligned}
N=\bar{N}= & m^{4} V T \times \frac{1}{4 \pi^{2}} \frac{e \bar{E}}{m^{2}} \frac{e \bar{B}}{m^{2}} \sum_{s= \pm 1} \sum_{n=0}^{\infty} \exp \left[-\pi \frac{m_{\perp}^{2}}{e \bar{E}}\right] \\
& \times\left[1+\mid \frac{\pi}{2} \frac{m_{\perp}^{2}}{e \bar{E}} \frac{\mathcal{E}_{0}}{\bar{E}}{ }_{1} \tilde{F}_{1}\left(1-\frac{i}{2} \frac{m_{\perp}^{2}}{e \bar{E}} ; 2 ; \frac{i \Omega^{2}}{2} \frac{{ }^{2} \bar{E}}{e}\right)\right] .
\end{aligned}
$$

Note that the phase $\phi$ is unimportant in the total production number $\stackrel{(-)}{N}(50)$, but can affect the distribution $\stackrel{(-)}{n}$ through the quantum interference between the Schwinger mechanism and one-photon pair production [74,75,87,98-100]. Below, we explicitly carry out the $n$ and $s$ summations in Eq. (50) to quantitatively discuss how the interplay and dynamical assistance between the Schwinger mechanism and one-photon pair production occur.

Figure 2 shows the total production number $N(50)$ as a function of the frequency of the perturbation $\Omega$ for various values of the magnetic field strength $e \bar{B}$. We confirm that the particle production is dominated by the Schwinger mechanism for small frequency $\Omega \lesssim 2 m$ and by one-photon pair production for large frequency $\Omega \gtrsim 2 m$ as we discussed analytically in Sec. II B 4. At the intermediate frequency $\Omega \sim$ $2 m$, the particle production becomes more abundant than what is expected from the Schwinger mechanism or one-photon pair production separately. This is the dynamical assistance between the two production mechanisms. Intuitively, the Schwinger mechanism is enhanced because the mass gap is reduced by the energy supply from the one-photon interaction and vice versa, as we explained in Fig. 1.

The frequency $\Omega$ dependence of one-photon pair production (i.e., the particle production for large frequency $\Omega \gtrsim 2 m$ ) dramatically changes with increasing magnetic field strength $e \bar{B}$. Namely, the production number as a function of $\Omega$ becomes flat (oscillating) if $e \bar{B}$ is small (large). This change occurs because the number of the Landau levels that contribute to the production changes depending on the size of $e \bar{B}$ (cf. Sec. II B 3). As shown in Fig. 3, the contribution from each Landau level $N_{n, s}$,

$$
N=\sum_{s} \frac{2 \pi}{L} \sum_{n} \int d p_{x} \int d p_{z} n_{p_{x}, p_{z}, n, s} \equiv \sum_{n, s} N_{n, s},
$$

always has an oscillating dependence on $\Omega$. This can be understood as an analog of the Franz-Keldysh oscillation in semiconductor physics [74,75,112,113], which occurs because of a quantum reflection in the presence of a tilted vacuum band structure under a strong electric field [74]. The quantum reflection is a counter phenomenon of the quantum tunneling responsible for the Schwinger mechanism. $N_{n, s}$ becomes significant above the energy threshold $\Omega \gtrsim 2 m_{\perp}=$ $2 \sqrt{m^{2}+e \bar{B}(2 n+1-s)}$. Therefore, many Landau levels can contribute to the production for small $e \bar{B}$, which cancels the oscillation of each contribution. On the other hand, such a cancellation does not take place for large $e \bar{B}$, for which only a small number of Landau levels can contribute to the production.

For very large magnetic field strength $e \bar{B} \gtrsim \Omega^{2}, m^{2}, e \bar{E}$, the lowest Landau level dominates the production $N \rightarrow N_{\mathrm{LLL}}$ [see Eq. (40)]. Then, the production number becomes just proportional to $e \bar{B}$ as shown in the small panels in Fig. 2 . The lowest Landau contribution $N_{\text {LLL }}$ becomes the largest at around the threshold value $\Omega \sim 2 m$, which is essential for the dynamical enhancement of chirality production, as we discuss in Sec. III.

Figure 4 shows the total production number $N$ (50) for various values of the electric field strength $e \bar{E}$. The behavior of the particle production dramatically changes depending 

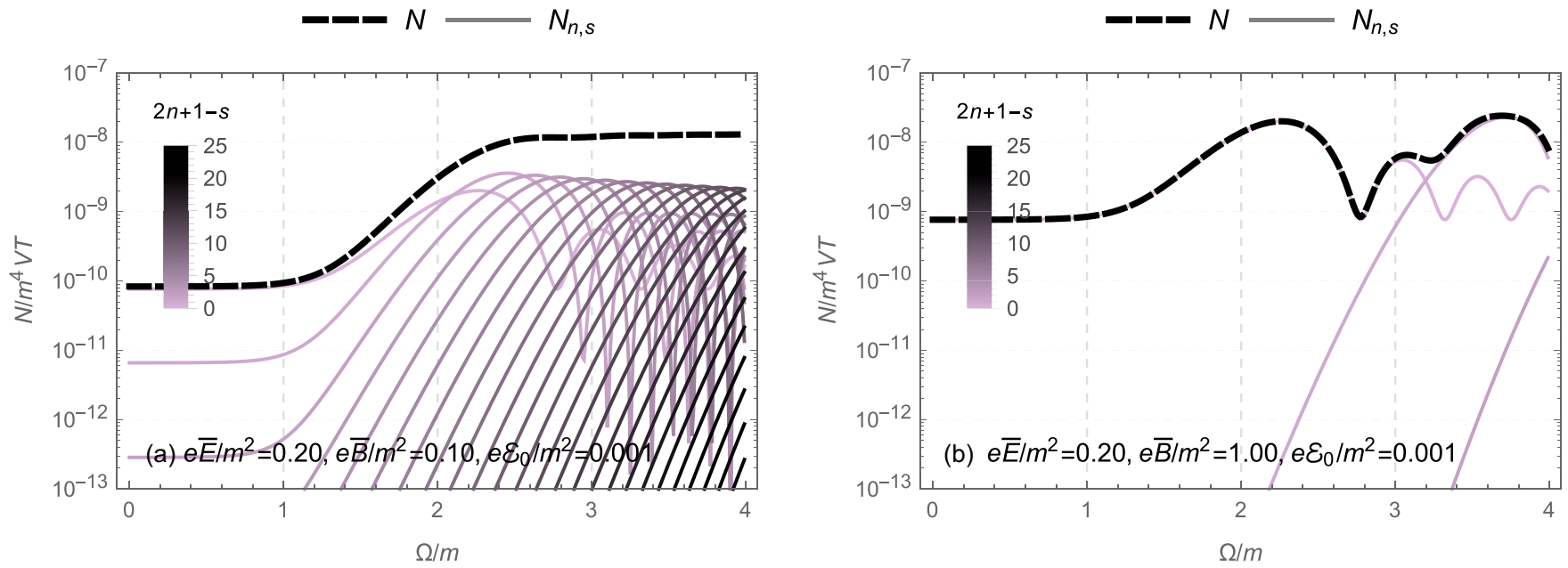

FIG. 3. The total production number $N=\sum_{n, s} N_{n, s}$ (50) (black dashed line) and the contribution from each Landau level $N_{n, s}$ (shaded lines) for various values of $2 n+1-s$ as a function of the frequency $\Omega / m$. The magnetic field strength $e \bar{B}$ is different between the two panels: $e \bar{B} / m^{2}=0.10$ (a) and 1.00 (b). The other parameters are fixed as $e \bar{E} / m^{2}=0.20$ and $e \mathcal{E}_{0} / m^{2}=0.001$.

on the size of $e \bar{E}$ (cf. Sec. II B 2). For a weak electric field $e \bar{E} \lesssim m^{2}, e \bar{B}$ (the left panel of Fig. 4), the interplay between the Schwinger mechanism and one-photon pair production occurs and is controlled by the size of the frequency $\Omega$. The particle production for large $\Omega$ shows up an oscillating behavior. For vanishing electric field strength $e \bar{E} / m^{2}=0$, the particle production for large $\Omega$ is dominated by onephoton pair production in the presence of a strong magnetic field (38), which is sharply peaked at the thresholds $\Omega=2 m_{\perp}$. The threshold behavior is smeared by the electric field $e \bar{E} \neq$ 0 since it supplies energy. For large electric field strength $e \bar{E} \gtrsim m^{2}, e \bar{B}$ (the right panel of Fig. 4), the production is dominated by the Schwinger mechanism no matter how large the frequency $\Omega$ is, and hence the production number becomes constant in $\Omega$. This is because the Schwinger mechanism becomes free from the exponential suppression and always surpasses one-photon pair production, which is suppressed by the power of $e \mathcal{E} / \mathrm{m}^{2}$. Note that the production number at $e \bar{E} / m^{2}=0.25$ [the lightest line in the panel (b) of Fig. 4] for large $\Omega$ does not coincide with the one-photon pair pro- duction formula (the dashed black line in the plot). This is because even though the electric field strength $e \bar{E} / m^{2}=0.25$ is still weak for the Schwinger mechanism to dominate the production for any values of $\Omega$, it is enough strong that the Schwinger mechanism becomes comparable to one-photon pair production. Thus, one cannot drop the contribution from the Schwinger mechanism as in the second line of Eq. (47), and the production number is given by the sum of the two mechanisms as $N=N_{\text {one-photon }}+N_{\text {Schwinger }}$.

\section{CHIRALITY PRODUCTION}

In this section, we discuss how the dynamically assisted Schwinger mechanism affects chirality production. Namely, we consider the slow strong parallel electromagnetic field superimposed by a fast weak perturbation (19) and derive an analytical formula for chirality production by explicitly evaluating an in-in vacuum expectation value of the chirality operator (see Sec. III A). Then, we use that formula to show that the dynamical assistance can enhance chirality production
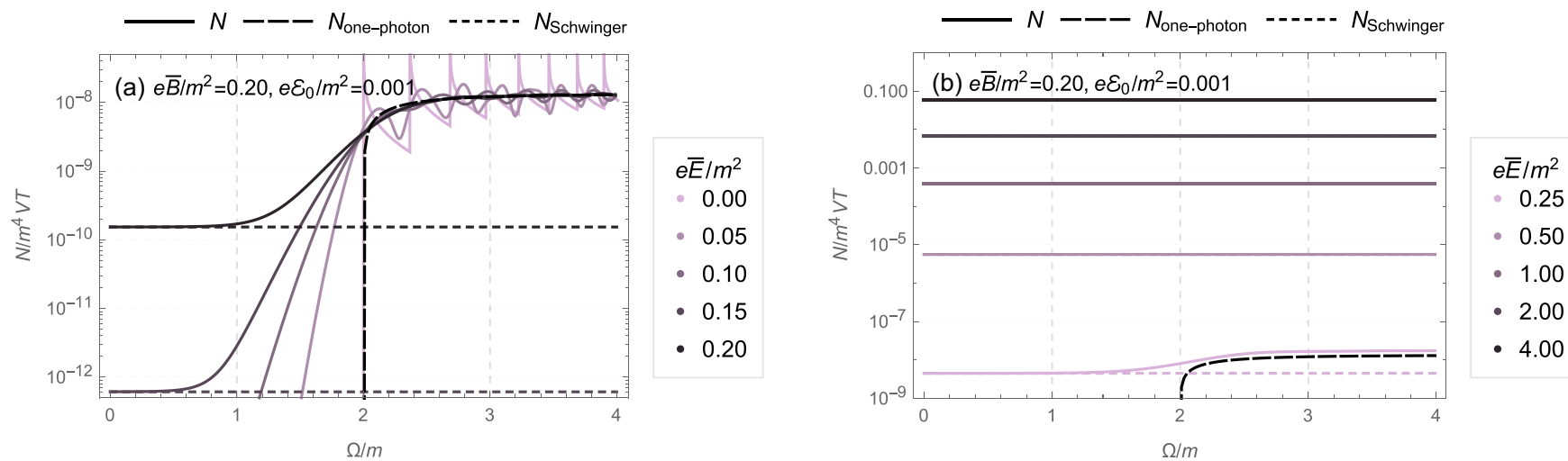

FIG. 4. The total production number $N$ (50) for the monochromatic perturbation (49) as a function of the frequency $\Omega / m$ for various values of the electric field strength $e \bar{E} / m^{2}=0.00,0.05,0.10,0.15,0.20$ (a) and $0.25,0.50,1.00,2.00,4.00$ (b). The other parameters are fixed as $e \bar{B} / m^{2}=0.20$ and $e \mathcal{E}_{0} / m^{2}=0.001$. For comparison, the one-photon pair production formula $N_{\text {one-photon }}$ (43) and the Schwinger formula $N_{\text {Schwinger }}(36)$ are plotted by the dashed and dotted lines, respectively. Note that the vertical scale is different between (a) and (b). 
by many orders of the magnitude, and that there exists an optimal frequency for a perturbation to maximize chirality production (see Sec. III B).

\section{A. Relation to the production number}

We analytically show that chirality production is related to the lowest Landau level production $N_{\mathrm{LLL}}$ and write down a formula for chiral charge produced from the vacuum in the presence of the slow strong parallel electromagnetic field superimposed by a perturbation (19) by using the production number formula derived in Sec. II.

The chiral charge at out state $Q_{5}$ produced from an initial vacuum state $\mid v a c ;$ in $\rangle$ is defined as

$$
Q_{5} \equiv \lim _{x^{0} \rightarrow \infty} \int d^{3} \boldsymbol{x}\left\langle\text { vac; in }\left|: \hat{\bar{\psi}}^{\text {(out) }} \gamma^{0} \gamma_{5} \hat{\psi}^{\text {(out) }}:\right| \text { vac; in }\right\rangle,
$$

where $\gamma_{5} \equiv i \gamma^{0} \gamma^{1} \gamma^{2} \gamma^{3}$ and $\langle: \cdots:\rangle$ is the normal ordering ${ }^{3}$ (e.g., $\left\langle: \hat{o}_{1} \hat{o}_{2}^{\dagger}:\right\rangle=-\left\langle\hat{o}_{2}^{\dagger} \hat{o}_{1}\right\rangle$ ), which is introduced to kill the ultraviolet divergence (i.e., subtract the unphysical vacuum contributions). Note that $Q_{5}$ is defined as an in-in expectation value, whose significance and relation to the in-out expectation value were clarified within the proper-time formalism in Ref. [114].

We can analytically evaluate Eq. (52). By substituting the mode expansion (29) into Eq. (52), we obtain

$$
\begin{aligned}
Q_{5}= & \frac{(2 \pi)^{3}}{V} \sum_{s} \frac{2 \pi}{L} \sum_{n} \int d p_{x} \int d p_{z} n_{p_{x}, p_{z}, n, s} \\
& \times \lim _{t \rightarrow \infty} \sum_{ \pm}( \pm 1)\left(\int d^{3} \boldsymbol{x}_{ \pm} \bar{\psi}_{p_{x}, p_{z}, n, s}^{\text {(out) }} \gamma^{0} \gamma_{5 \pm} \psi_{p_{x}, p_{z}, n, s}^{\text {(out) }}\right),
\end{aligned}
$$

where we used $\quad n_{p_{x}, p_{z}, n, s}=\bar{n}_{-p_{x},-p_{z}, n,-s} \quad$ and dropped a severely oscillating factor $\lim _{x^{0} \rightarrow \infty-}$ $\bar{\psi}_{p_{x}, p_{z}, n, s}^{\text {(out) }} \gamma^{0} \gamma_{5+} \psi_{p_{x}, p_{z}, n, s}^{\text {(out) }} \propto \lim _{x^{0} \rightarrow \infty} e^{-2 i \sqrt{m_{\perp}^{2}+p_{z}^{2}} x^{0}} \rightarrow 0$ because of the $i \epsilon$ prescription of quantum field theory. Then, by using the analytical expression for the mode function ${ }_{ \pm} \psi_{p_{x}, p_{z}, n, s}^{(\text {as })}(21)$, we can explicitly evaluate the matrix element in Eq. (53), and the result reads

$$
Q_{5}=\sum_{s} \sum_{n} N_{n, s} \times 2 s \frac{m^{2}}{m_{\perp}^{2}}=2 N_{\mathrm{LLL}} .
$$

To get the second equality, we used $N_{n, s=-1}=N_{n+1, s=+1}$. The higher Landau level contributions cancel each other, and we are left only with the lowest Landau level contribution $2 N_{\text {LLL }}=N_{n=0, s=+1}+\bar{N}_{n=0, s=-1}$. Equation (54) suggests that chirality production should be enhanced if the lowest Landau level production $N_{\mathrm{LLL}}$ is enhanced by, e.g., the dynamically assisted Schwinger mechanism.

Within the perturbation theory in the Furry picture (see Sec. II A), one can explicitly evaluate $N_{\mathrm{LLL}}$ as Eq. (40).

\footnotetext{
${ }^{3}$ Precisely speaking, normal ordering with respect to "out-state" operators.
}

Therefore, we have

$$
\begin{aligned}
Q_{5}= & Q_{5}(\mathcal{E}=0) \\
\times & {\left[1+\frac{2 \pi}{T}\left\{\frac{1}{2} \frac{m^{2}}{e \bar{E}} \frac{\tilde{\mathcal{E}}(0)}{\bar{E}}+\frac{1}{4}\left(\frac{m^{2}}{e \bar{E}}\right)^{2} \int_{0}^{\infty} d \omega\right.\right.} \\
& \left.\left.\times\left|\frac{\tilde{\mathcal{E}}(\omega)}{\bar{E}}{ }_{1} \tilde{F}_{1}\left(1-\frac{i}{2} \frac{m^{2}}{e \bar{E}} ; 2 ; \frac{i}{2} \frac{\omega^{2}}{e \bar{E}}\right)\right|^{2}\right\}\right],
\end{aligned}
$$

where

$$
Q_{5}(\mathcal{E}=0)=V T \times \frac{e \bar{E} e \bar{B}}{2 \pi^{2}} \exp \left[-\pi \frac{m^{2}}{e \bar{E}}\right]
$$

is chirality production without any perturbations $[63,114,115] . Q_{5}(\mathcal{E}=0)$ is exponentially suppressed by the mass $m$, and hence chirality production for massive particles is usually negligible. However, the dynamical assistance by a perturbation,

$$
\frac{Q_{5}(\mathcal{E})}{Q_{5}(\mathcal{E}=0)}-1>0,
$$

is always positive and, as we shall demonstrate below, can enhance chirality production by many orders of the magnitude. Hence, a sizable amount of chirality can be produced even for massive particles. Note that in the massless limit $m \rightarrow 0$ the dynamical assistance goes away because the Schwinger mechanism dominates the production, and we have

$$
Q_{5} \stackrel{m \rightarrow 0}{\longrightarrow} Q_{5}(\mathcal{E}=0)=V T \times \frac{e \bar{E} e \bar{B}}{2 \pi^{2}},
$$

which is consistent with the Adler-Bell-Jackiw (ABJ) anomaly relation for $m=0: \partial_{t}\left(Q_{5} / V\right)=e \bar{E} e \bar{B} / 2 \pi^{2}$ [56,57]. Equation (58) means that the dynamical assistance is not important for chirality production for light particles whose mass is sufficiently lighter than the electric field strength $e \bar{E}$.

\section{B. The dynamical assistance to chirality production}

We evaluate the formula (55) with the monochromatic perturbation (49) to quantitatively discuss how the dynamical assistance modifies chirality production. We display the result in Fig. 5.

The dynamical assistance by a perturbation can significantly enhance chirality production for massive particles. The size of the enhancement strongly depends on the frequency of a perturbation $\Omega$. For frequencies below the mass gap $\Omega \ll 2 m$, the enhancement is not significant and chirality production is exponentially suppressed by the mass per Eq. (56). The enhancement becomes the maximum at around the energy threshold for the lowest Landau level $\Omega \sim 2 m$. This is because the dynamically assisted Schwinger mechanism takes place, i.e., the threshold energy is effectively reduced by the energy supply by a perturbation and thus the Schwinger mechanism for the lowest Landau level is enhanced, which results in the enhancement of chirality production. Above the energy threshold $\Omega \gtrsim 2 m$, the enhancement decays slowly and shows an oscillating dependence on $\Omega$, which can be understood as an analog of the Franz-Keldysh oscillation [74,75,112,113]. Note that the enhancement increases quadratically with $e \mathcal{E}_{0}$ and that the $\Omega$ dependence is unchanged with $e \mathcal{E}_{0}$ unless $e \mathcal{E}_{0}$ 


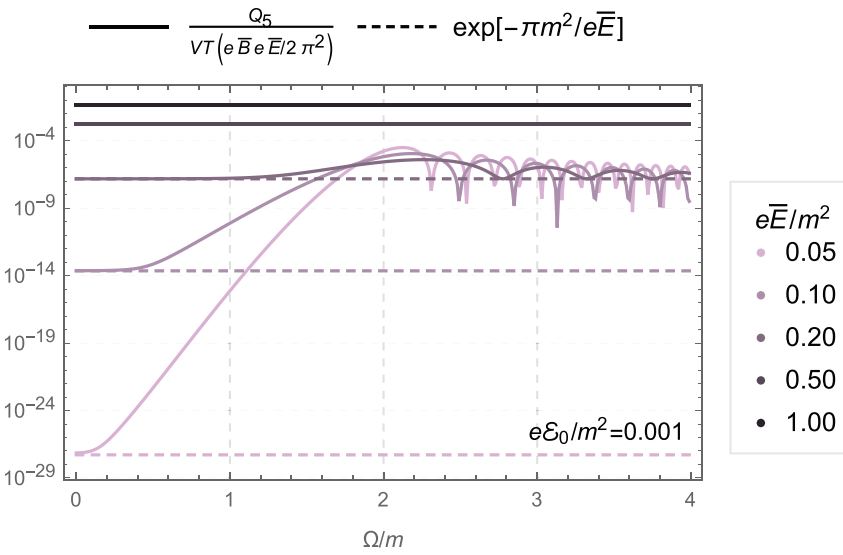

FIG. 5. Chirality production $Q_{5}$ (55) scaled by the anomaly factor $e \bar{E} e \bar{B} / 2 \pi^{2}$ for the monochromatic perturbation (49) as a function of the frequency $\Omega / m$. Different shadings distinguish electric field strengths $e \bar{E} / m^{2}=0.05,0.10,0.20,0.50$, and 1.00 . The dashed lines represent the mass suppression without perturbations (56). The strength of the perturbative electric field is fixed as $e \mathcal{E}_{0} / m^{2}=$ 0.001 . Note that the result is independent of the magnetic field strength $e \bar{B} / m^{2}$.

becomes so large that the formula (55), which only takes into account the lowest correction from a perturbation, becomes invalid.

The enhancement is not significant for light particles, as we discussed below Eq. (58). This is because chirality production is free from the exponential suppression for light particles, and hence the dynamical assistance, which is always suppressed by powers of the mass, can only give a secondary contribution. Note that the insensitivity to the time dependence of a field for chirality production for light particles and the decrease of that for massive particles for a slower field configuration are consistent with Ref. [110], in which chirality production for massless and massive particles by a pulsed electric field with a sudden switching on/off was discussed.

\section{SUMMARY AND DISCUSSION}

We have studied particle production from the vacuum by a slow strong parallel electromagnetic field superimposed by a fast weak perturbation. We have generalized the perturbation theory in the Furry picture [74,75,84-89] to include a magnetic component in the slow strong field configuration and derived an analytical formula for the production number. Based on the formula, we have analytically discussed the particle production, focusing on the interplay between the Schwinger mechanism and one-photon pair production and the dynamical assistance between the two production mechanisms. In particular, we have shown that (i) the strength of the strong electric field and the frequency of a perturbation control the interplay, and the Schwinger mechanism (onephoton pair production) dominates the particle production when the electric field strength is large (small) and the frequency is small (large); (ii) the two production mechanisms occur at the same time and assist each other at intermediate values of the electric field strength and the frequency, and the particle production is significantly enhanced by many orders of magnitude (i.e., the dynamically assisted Schwinger mechanism [67-71]); (iii) the effect of a strong magnetic field is to discretize the energy level through the Landau quantization, which enhances the production proportionally to the magnetic field strength because of the enhancement of the phase space, and results in sharp threshold behaviors in the production number when the magnetic field strength is comparable to or stronger than the electric one; and (iv) the lowest Landau approximation becomes valid for a very strong magnetic field compared to mass and the electric field strength, for which the production number is maximized at frequency around the energy threshold $\Omega \sim 2 m$ and exhibits oscillating dependence on $\Omega$ for $\Omega \gtrsim 2 m$ (an analog of the Franz-Keldysh oscillation [74,75,112,113]).

We also have clarified how the dynamical assistance to the particle production affects chirality production. We have explicitly evaluated the in-in vacuum expectation value of the chirality operator and shown that chirality production is determined solely by the lowest Landau level production. Therefore, chirality production is enhanced because the lowest Landau level production is enhanced by the dynamical assistance. We have written down an analytical formula for chirality production based on the production number formula within the perturbation theory in the Furry picture, and shown that (i) chirality production for light particles whose mass is lighter than the electric field strength is less affected by the dynamical assistance; (ii) chirality production for massive particles is significantly enhanced by many orders of the magnitude; and (iii) the enhancement becomes the largest at frequency $\Omega \sim 2 m$, where the dynamical assistance to the lowest Landau level production is maximized.

An interesting application of our results is in heavy-ion collisions at RHIC and the LHC. We have shown in the present paper that perturbations on top of a slow strong parallel electromagnetic field significantly affect particle and chirality production. In heavy-ion collisions, the glasma [47-51] plays the role of a slow strong parallel (chromo)electromagnetic field and a weak fast perturbation is naturally seeded by quark/gluon jets produced by initial hard collisions. Therefore, we expect that particle and chirality production in heavyion collisions should be modified significantly by jets on top of the glasma. For example, a sizable amount of chirality may be produced even for massive strange and charm quarks because of the dynamical assistance by jets. This could leave experimental signatures such as charge asymmetry of heavy hadrons through the chiral magnetic effect. Another example is that the enhancement of the particle production by jets may speed up the formation of the quark-gluon plasma in heavy-ion collisions, which could be interesting to the early thermalization/hydrodynamization puzzle [116,117]. We may also expect that propagation of jets should be modified significantly by the glasma because jets lose their energy via the particle production in the glasma. This could give an additional contribution to the jet quenching and broadening phenomena.

Another interesting application is in intense laser experiments at, e.g., ELI and XCELS. Experimentally, one may realize a parallel slow strong electromagnetic field configuration by colliding two counterpropagating laser beams with different polarization. For example, by colliding two laser beams 
described by gauge potentials $\overline{\boldsymbol{A}}_{1}=(a / k) \sin \left[k\left(x^{1}-x^{0}\right)\right] \times$ $(0,1,0)$ and $\overline{\boldsymbol{A}}_{2}=(a / k) \sin \left[k\left(-x^{1}-x^{0}\right)\right] \times(0, \sin \phi, \cos \phi)$ and assuming that the laser beams are sufficiently slow $|k| \ll$ 1 and that the particle production occurs at the region $\left|k x^{1}\right| \ll$ 1 , one gets

$$
\overline{\boldsymbol{E}} \sim a\left(\begin{array}{c}
0 \\
1+\sin \phi \\
\cos \phi
\end{array}\right), \quad \overline{\boldsymbol{B}} \sim \frac{\cos \phi}{1+\sin \phi} \overline{\boldsymbol{E}} .
$$

Thus, we have $\overline{\boldsymbol{E}} \| \overline{\boldsymbol{B}}$ and can control the relative size between the electric and magnetic field strengths by tuning the polarization angle $\phi$ (e.g., $\bar{E}=\bar{B} \neq 0$ for $\phi=0 ; \bar{E}=0, \bar{B} \neq 0$ for $\phi=-\pi / 2$; and $\bar{E} \neq 0, \bar{B}=0$ for $\phi=\pi / 2$ ). As shown in the present paper, the existence of a parallel strong magnetic field makes the particle production phenomenologically richer, e.g., it results in the nontrivial frequency dependence for a high-frequency perturbation and chirality production. The sharp frequency dependence for strong $e \bar{B}$ may be detected by using techniques of modulation spectroscopy [118], which is a well-established method to measure the FranzKeldysh oscillation in semiconductor physics. Observing chirality production is not only new to laser physics, but also important to understand the chirality production mechanism in heavy-ion collisions and consequent observables, which are less understood in the heavy-ion community despite great experimental/theoretical efforts over the past decade. Also, chirality production may induce anomalous transport phenomena such as the chiral magnetic effect even in laser systems. This is an opportunity to study anomalous transport phenomena and may open up a new connection between laser physics and the other areas of physics since anomalous transport phenomena have been attracting attention from broad areas of physics with different energy scales, such as Weyl/Dirac semi-metals, heavy-ion collisions, neutron stars, and supernovae.

For future work, it is interesting to extend our lowest order formula $(34)$ by including higher order $e^{k}(k \geqslant 2)$ corrections. This enables us to discuss the dynamical assistance not only by one-photon pair production $\left(\gamma+\bar{E}, \bar{B} \rightarrow e^{+} e^{-}\right)$, but also by multiphoton pair production $\left(n \gamma+\bar{E}, \bar{B} \rightarrow e^{+} e^{-}\right)$ and multi-pair production by a photon $\left(\gamma+\bar{E}, \bar{B} \rightarrow n e^{+} e^{-}\right)$, which are parametrically suppressed by the strength of a perturbation as $\left(e \mathcal{E} / m^{2}\right)^{2 n}$ but become important if $e \mathcal{E}$ becomes strong. Such higher order processes may, for example, induce additional peak structures in the production number as a function of the frequency of a perturbation $[75,119$ 122]. Another possible extension is to consider a perturbation with various polarizations. Not only the production number/distribution, but also spin dynamics is modified by polarization, which results in nontrivial spin accumulation and/or generation of a spin current [43,75,123,124]. Non trivial spin dependence/observables imply a modification to chirality production and, therefore, may induce novel chiralitydependent observables. It is also important to understand polarization effects to discuss heavy-ion collisions since jets in heavy-ion collisions are not necessarily longitudinal with respect to the glasma. It is very straightforward to extend our formalism to such a general perturbation with different polarization as well as, for example, with spatial dependence, which has been discussed within the worldline instanton formalism for a slowly varying case $[105,106]$ and within a numerical method $[107,108]$. The last direction that we would like to mention is to consider different types of perturbations. In the present paper, we concentrated on a perturbation by a weak fast electromagnetic field (or a dynamical photon). In principle, any kinds of perturbations (or, generally, external forces) may dynamically assist the Schwinger mechanism and vice versa as long as they supply energy to the vacuum. Such a consideration has been done recently for vibrating plates (or the dynamical Casimir effect) [125]. A strong magnetic field drastically changes the dimensionality of the system because of the Landau quantization. Hence, the dynamical assistance by geometric perturbations such as vibration of plates and gravitational fields should be modified.

\section{ACKNOWLEDGMENTS}

The author would like to thank Gerald V. Dunne, Kenji Fukushima, Koichi Hattori, and Xu-Guang Huang for fruitful discussions. This work was partially supported by National Natural Science Foundation in China (NSFC) under Grant No. 11847206.
[1] F. Gelis and N. Tanji, Schwinger mechanism revisited, Prog. Part. Nucl. Phys. 87, 1 (2015).

[2] R. Ruffini, G. Vereshchagin, and S.-S. Xue, Electron-positron pairs in physics and astrophysics: From heavy nuclei to black holes, Phys. Rep. 487, 1 (2010).

[3] G. V. Dunne, Heisenberg-Euler effective Lagrangians: Basics and extensions, in From Fields to Strings, edited by M. Shifman et al. (World Scientific, Singapore, 2005), Vol. 1, p. 445.

[4] F. Sauter, Ueber das verhalten eines elektrons im homogenen elektrischen feld nach der relativistischen theorie Diracs, Z. Phys. 69, 742 (1931).

[5] W. Heisenberg and H. Euler, Folgerungen aus der Diracschen theorie des positrons, Z. Phys. 98, 714 (1936).

[6] J. Schwinger, On Gauge Invariance and Vacuum Polarization, Phys. Rev. 82, 664 (1951).
[7] L. Landau, Zur Theorie der Energieubertragung. II, Phys. Z. Sowjetunion 2, 46 (1932).

[8] C. Zener, Non-adiabatic crossing of energy levels, Proc. R. Soc. London Ser. A 137, 696 (1932).

[9] E. C. G. Stueckelberg, Theorie der unelastischen Stosse zwischen Atomen, Helv. Phys. Acta. 5, 369 (1932).

[10] E. Majorana, Atomi orientati in campo magnetico variabile, Nuovo Cimento 9, 43 (1932).

[11] S. P. Gavrilov and D. M. Gitman, Vacuum instability in external fields, Phys. Rev. D 53, 7162 (1996).

[12] M. S. Marinov and V. S. Popov, Pair production in an electromagnetic field (case of arbitrary spin), Sov. J. Nucl. Phys. 15, 702 (1972).

[13] Y. Hidaka, T. Iritani, and H. Suganuma, Fast vacuum decay into quark pairs in strong color electric and magnetic fields, in International Conference on the Structure of Bbaryons 
(Baryons '10), 7-11 December 2010, Osaka, edited by A. Hosaka, K. Khemchandani, H. Nagahiro, and K. Nawa, AIP Conf. Proc. No. 1388 (AIP, New York, 2011), p. 516.

[14] Y. Hidaka, T. Iritani, and H. Suganuma, Fast vacuum decay into particle pairs in strong electric and magnetic fields, arXiv:1102.0050.

[15] K. Hattori, K. Itakura, and S. Ozaki, Note on all-order Landaulevel structures of the Heisenberg-Euler effective actions for QED and QCD, arXiv:2001.06131.

[16] A. I. Nikishov, Pair production by a constant external field, Sov. Phys. JETP 30, 660 (1970).

[17] N. Tanji, Dynamical view of pair creation in uniform electric and magnetic fields, Ann. Phys. (NY) 324, 1691 (2009).

[18] V. S. Popov, Pair production in a variable external field (quasiclassical approximation), Sov. Phys. JETP 34, 709 (1972).

[19] N. Tanji, Quark pair creation in color electric fields and effects of magnetic fields, Ann. Phys. (N.Y. ) 325, 2018 (2010).

[20] X.-L. Sheng, R.-H. Fang, Q. Wang, and D. H. Rischke, Wigner function and pair production in parallel electric and magnetic fields, Phys. Rev. D 99, 056004 (2019).

[21] A. Iwazaki, Pair creation in electric flux tube and chiral anomaly, Phys. Rev. C 80, 052202(R) (2009).

[22] D. Karabali, S. Kurkcuoglu, and V. P. Nair, Magnetic field and curvature effects on pair production I: Scalars and spinors, Phys. Rev. D 100, 065005 (2019).

[23] D. Karabali, S. Kurkcuoglu, and V. P. Nair, Magnetic field and curvature effects on pair production II: Vectors and implications for chromodynamics, Phys. Rev. D 100, 065006 (2019).

[24] N. Tanji and K. Itakura, Schwinger mechanism enhanced by the Nielsen-Olesen instability, Phys. Lett. B 713, 117 (2012).

[25] N. K. Nielsen and P. Olesen, An unstable Yang-Mills field mode, Nucl. Phys. B 144, 376 (1978).

[26] N. K. Nielsen and P. Olesen, Electric vortex lines from the Yang-Mills theory, Phys. Lett. B 79, 304 (1978).

[27] S. J. Chang and N. Weiss, Instability of constant Yang-Mills fields, Phys. Rev. D 20, 869 (1979).

[28] T. Kobayashi and N. Afshordi, Schwinger effect in 4D de Sitter space and constraints on magnetogenesis in the early universe, J. High Energy Phys. 10 (2014) 166.

[29] S. Shakeri, M. Ali Gorji, and H. Firouzjahi, Schwinger mechanism during inflation, Phys. Rev. D 99, 103525 (2019).

[30] O. O. Sobol, E. V. Gorbar, M. Kamarpour, and S. I. Vilchinskii, Influence of backreaction of electric fields and Schwinger effect on inflationary magnetogenesis, Phys. Rev. D 98, 063534 (2018).

[31] R. Sharma, S. Jagannathan, T. R. Seshadri, and K. Subramanian, Challenges in inflationary magnetogenesis: Constraints from strong coupling, backreaction, and the Schwinger effect, Phys. Rev. D 96, 083511 (2017).

[32] C. Stahl, Schwinger effect impacting primordial magnetogenesis, Nucl. Phys. B 939, 95 (2019).

[33] T. Kobayashi and M. S. Sloth, Early cosmological evolution of primordial electromagnetic fields, Phys. Rev. D 100, 023524 (2019).

[34] V. Domcke, Y. Ema, and K. Mukaida, Chiral anomaly, Schwinger effect, Euler-Heisenberg Lagrangian, and application to axion inflation, J. High Energy Phys. 02 (2020) 055
[35] T. Oka and H. Aoki, Nonequilibrium quantum breakdown in a strongly correlated electron system, in Quantum and Semiclassical Percolation and Breakdown in Disordered Solids, edited by A. K. Sen, K. K. Bardhan, and B. K. Chakrabarti, Lecture Notes in Physics Vol. 762 (Springer-Verlag, Berlin, 2008).

[36] D. Allor, T. D. Cohen, and D. A. McGady, The Schwinger mechanism and graphene, Phys. Rev. D 78, 096009 (2008).

[37] F. Fillion-Gourdeau and S. MacLean, Time-dependent pair creation and the Schwinger mechanism in graphene, Phys. Rev. B 92, 035401 (2015).

[38] V. Kasper, F. Hebenstreit, M. K. Oberthaler, and J. Berges, Schwinger pair production with ultracold atoms, Phys. Lett. B 760, 742 (2016).

[39] V. Kasper, F. Hebenstreit, F. Jendrzejewski, M. K. Oberthaler, and J. Berges, Implementing quantum electrodynamics with ultracold atomic systems, New J. Phys. 19, 023030 (2017).

[40] N. Szpak and R. Schutzhold, Optical lattice quantum simulator for QED in strong external fields: Spontaneous pair creation and the Sauter-Schwinger effect, New J. Phys. 14, 035001 (2012).

[41] A. M. Piñeiro, D. Genkina, M. Lu, and I. B. Spielman, SauterSchwinger effect with a quantum gas, New J. Phys. 21, 083035 (2019).

[42] A. G. Green and S. L. Sondhi, Nonlinear Quantum Critical Transport and the Schwinger Mechanism for a SuperfluidMott-Insulator Transition of Bosons, Phys. Rev. Lett. 95, 267001 (2005).

[43] X.-G. Huang, M. Matsuo, and H. Taya, Spontaneous generation of spin current from the vacuum by strong electric fields, Prog. Theor. Exp. Phys. 2019, 113B02 (2019).

[44] J. A. Grifols, E. Masso, and S. Mohanty, Production of light pseudoscalars in external electromagnetic fields by the Schwinger mechanism, Phys. Rev. D 65, 055004 (2002).

[45] J. A. Grifols, E. Masso, S. Mohanty, and K. V. Shajesh, Pair production of light pseudoscalar particles in strong inhomogeneous fields by the Schwinger mechanism, Phys. Rev. D 60, 097701 (1999).

[46] X.-G. Huang, D. E. Kharzeev, and H. Taya, Real-time dynamics of axion particle production due to spontaneous decay of a coherent axion field, Phys. Rev. D 101, 016011 (2020).

[47] A. Kovner, L. McLerran, and H. Weigert, Gluon production at high transverse momentum in the McLerran-Venugopalan model of nuclear structure functions, Phys. Rev. D 52, 3809 (1995).

[48] A. Kovner, L. McLerran, and H. Weigert, Gluon production from non-Abelian Weizsäcker-Williams fields in nucleusnucleus collisions, Phys. Rev. D 52, 6231 (1995).

[49] T. Lappi and L. McLerran, Some features of the glasma, Nucl. Phys. A 772, 200 (2006).

[50] F. E. Low, Model of the bare Pomeron, Phys. Rev. D 12, 163 (1975).

[51] S. Nussinov, Colored-Quark Version of Some Hadronic Puzzles, Phys. Rev. Lett. 34, 1286 (1975).

[52] N. K. Glendenning and T. Matsui, Creation of $q \bar{q}$ pairs in a chromoelectric flux tube, Phys. Rev. D 28, 2890 (1983).

[53] K. Kajantie and T. Matsui, Decay of strong color electric field and thermalization in ultra-relativistic nucleus-nucleus collisions, Phys. Lett. B 164, 373 (1985). 
[54] G. Gatoff, A. K. Kerman, and T. Matsui, Flux-tube model for ultrarelativistic heavy-ion collisions: Electrohydrodynamics of a quark-gluon plasma, Phys. Rev. D 36, 114 (1987).

[55] H. Taya, Quark and gluon production from a boost-invariantly expanding color electric field, Phys. Rev. D 96, 014033 (2017).

[56] S. L. Adler, Axial-Vector Vertex in Spinor Electrodynamics, Phys. Rev. 177, 2426 (1969).

[57] J. S. Bell and R. Jackiw, A PCAC puzzle: $\pi^{0} \rightarrow \gamma \gamma$ in the $\sigma$-model, Nuovo Cimento A 60, 47 (1969).

[58] H. B. Nielsen and Masao Ninomiya, The Adler-Bell-Jackiw anomaly and Weyl fermions in a crystal, Phys. Lett. B 130, 389 (1983)

[59] D. E. Kharzeev, The chiral magnetic effect and anomalyinduced transport, Prog. Part. Nucl. Phys. 75, 133 (2014).

[60] X.-G. Huang, Electromagnetic fields and anomalous transports in heavy-ion collisions-A pedagogical review, Rep. Prog. Phys. 79, 076302 (2016).

[61] D. E. Kharzeev, L. D. McLerran, and H. J. Warringa, The Effects of topological charge change in heavy ion collisions: "Event by event $\mathcal{P}$ and $\mathcal{C P}$ violation", Nucl. Phys. A 803, 227 (2008).

[62] K. Fukushima, D. E. Kharzeev, and H. J. Warringa, The chiral magnetic effect, Phys. Rev. D 78, 074033 (2008).

[63] K. Fukushima, D. E. Kharzeev, and H. J. Warringa, Real-Time Dynamics of the Chiral Magnetic Effect, Phys. Rev. Lett. 104, 212001 (2010).

[64] J. Zhao and F. Wang, Experimental searches for the chiral magnetic effect in heavy-ion collisions, Prog. Part. Nucl. Phys. 107, 200 (2019).

[65] V. Yanovsky et al., Ultra-high intensity $300 \mathrm{TW}$ laser at $0.1 \mathrm{~Hz}$ repetition rate, Opt. Express 16, 2109 (2008).

[66] A. Di Piazza, C. Muller, K. Z. Hatsagortsyan, and C. H. Keitel, Extremely high-intensity laser interactions with fundamental quantum systems, Rev. Mod. Phys. 84, 1177 (2012).

[67] R. Schutzhold, H. Gies, and G. Dunne, Dynamically Assisted Schwinger Mechanism, Phys. Rev. Lett. 101, 130404 (2008).

[68] A. Di Piazza, E. Lotstedt, A. I. Milstein, and C. H. Keitel, Barrier Control in Tunneling $e^{+}-e^{-}$Photoproduction, Phys. Rev. Lett. 103, 170403 (2009).

[69] G. V. Dunne, H. Gies, and R. Schutzhold, Catalysis of Schwinger vacuum pair production, Phys. Rev. D 80, 111301(R) (2009).

[70] A. Monin and M. B. Voloshin, Photon-stimulated production of electron-positron pairs in electric field, Phys. Rev. D 81, 025001 (2010).

[71] A. Monin and M. B. Voloshin, Semiclassical calculation of photon-stimulated Schwinger pair creation, Phys. Rev. D 81, 085014 (2010)

[72] V. W. Franz, Einfluss eines elektrischen Feldes auf eine optische Absorptionskante, Z. Naturforsch. Teil A 13, 484 (1958).

[73] L. V. Keldysh, The effect of a strong electric field on the optical properties of insulating crystals, Sov. Phys. JETP 7, 788 (1958).

[74] H. Taya, Franz-Keldysh effect in strong-field QED, Phys. Rev. D 99, 056006 (2019).

[75] X.-G. Huang and H. Taya, Spin-dependent dynamically assisted Schwinger mechanism, Phys. Rev. D 100, 016013 (2019).
[76] E. Brezin and C. Itzykson, Pair production in vacuum by an alternating field, Phys. Rev. D 2, 1191 (1970).

[77] L. V. Keldysh, Ionization in the field of a strong electromagnetic wave, JETP 20, 1307 (1965).

[78] H. Taya, H. Fujii, and K. Itakura, Finite pulse effects on $e^{+} e^{-}$ pair creation from strong electric fields, Phys. Rev. D 90, 014039 (2014).

[79] G. V. Dunne and C. Schubert, Worldline instantons and pair production in inhomogeneous fields, Phys. Rev. D 72, 105004 (2005).

[80] G. V. Dunne, Q. H. Wang, H. Gies, and C. Schubert, Worldline instantons II: The fluctuation prefactor, Phys. Rev. D 73, 065028 (2006).

[81] G. V. Dunne and Q. H. Wang, Multidimensional worldline instantons, Phys. Rev. D 74, 065015 (2006).

[82] S. A. Smolyansky, G. Roepke, S. Schmidt, D. Blaschke, V. D. Toneev, and A. V. Prozorkevich, Dynamical derivation of a quantum kinetic equation for particle production in the Schwinger mechanism, arXiv:hep-ph/9712377.

[83] S. M. Schmidt, D. Blaschke, G. Ropke, S. A. Smolyansky, A. V. Prozorkevich, and V. D. Toneev, A Quantum kinetic equation for particle production in the Schwinger mechanism, Int. J. Mod. Phys. E 7, 709 (1998).

[84] W. H. Furry, On Bound State and Scattering in Positron Theory, Phys. Rev. 81, 115 (1951).

[85] E. S. Fradkin and D. M. Gitman, Furry picture for quantum electrodynamics with pair creating external field, Fortschr. Phys. 29, 381 (1981).

[86] E. S. Fradkin, D. M. Gitman, and S. M. Shvartsman, Quantum Electrodynamics with Unstable Vacuum (Springer-Verlag, Berlin, 1991).

[87] G. Torgrimsson, C. Schneider, and R. Schutzhold, Dynamically assisted Sauter-Schwinger effect - non-perturbative versus perturbative aspects, J. High Energy Phys. 06 (2017) 043.

[88] G. Torgrimsson, Perturbative methods for assisted nonperturbative pair production, Phys. Rev. D 99, 096002 (2019).

[89] G. Torgrimsson, C. Schneider, and R. Schutzhold, SauterSchwinger pair creation dynamically assisted by a plane wave, Phys. Rev. D 97, 096004 (2018).

[90] F. Fillion-Gourdeau, F. Hebenstreit, D. Gagnon, and S. MacLean, Pulse shape optimization for electron-positron production in rotating fields, Phys. Rev. D 96, 016012 (2017).

[91] M. F. Linder, C. Schneider, J. Sicking, N. Szpak, and R. Schutzhold, Pulse shape dependence in the dynamically assisted Sauter-Schwinger effect, Phys. Rev. D 92, 085009 (2015).

[92] F. Hebenstreit and F. Fillion-Gourdeau, Optimization of Schwinger pair production in colliding laser pulses, Phys. Lett. B 739, 189 (2014).

[93] S. Dong, J. Unger, J. Bryan, Q. Su, and R. Grobe, Symbiotic versus nonsymbiotic optimization for spatial and temporal degrees of freedom in pair creation, Phys. Rev. E 101, 013310 (2020).

[94] C. Kohlfurst, M. Mitter, G. von Winckel, F. Hebenstreit, and R. Alkofer, Optimizing the pulse shape for Schwinger pair production, Phys. Rev. D 88, 045028 (2013).

[95] N. Abdukerim, Z.-L. Li, and B.-S. Xie, Effects of laser pulse shape and carrier envelope phase on pair production, Phys. Lett. B 726, 820 (2013). 
[96] M. Orthaber, F. Hebenstreit, and R. Alkofer, Momentum spectra for dynamically assisted Schwinger pair production, Phys. Lett. B 698, 80 (2011).

[97] C. Fey and R. Schutzhold, Momentum dependence in the dynamically assisted Sauter-Schwinger effect, Phys. Rev. D 85, 025004 (2012).

[98] C. K. Dumlu and G. V. Dunne, The Stokes Phenomenon and Schwinger Vacuum Pair Production in Time-Dependent Laser Pulses, Phys. Rev. Lett. 104, 250402 (2010).

[99] C. K. Dumlu and G. V. Dunne, Complex worldline instantons and quantum interference in vacuum pair production, Phys. Rev. D. 84, 125023 (2011).

[100] F. Hebenstreit, R. Alkofer, G. V. Dunne, and H. Gies, Momentum Signatures for Schwinger Pair Production in Short Laser Pulses with a Subcycle Structure, Phys. Rev. Lett. 102, 150404 (2009).

[101] A. D. Panferov, S. A. Smolyansky, A. Otto, B. Kaempfer, D. Blaschke, and L. Juchnowski, Assisted dynamical Schwinger effect: Pair production in a pulsed bifrequent field, Eur. Phys. J. D 70, 56 (2016).

[102] Z. L. Li, D. Lu, B. S. Xie, L. B. Fu, J. Liu, and B. F. Shen, Enhanced pair production in strong fields by multipleslit interference effect with dynamically assisted Schwinger mechanism, Phys. Rev. D 89, 093011 (2014).

[103] M. Ababekri, B.-S. Xie, and J. Zhang, Effects of finite spatial extent on Schwinger pair production, Phys. Rev. D 100, 016003 (2019).

[104] M. Ababekri, S. Dulat, B. S. Xie, and J. Zhang, Chirp effects on pair production in oscillating electric fields with spatial inhomogeneity, arXiv:1912.05302.

[105] C. Schneider and R. Schutzhold, Dynamically assisted SauterSchwinger effect in inhomogeneous electric fields, J. High Energy Phys. 02 (2016) 164.

[106] P. Copinger and K. Fukushima, Spatially Assisted Schwinger Mechanism and Magnetic Catalysis, Phys. Rev. Lett. 117, 081603 (2016).

[107] I. A. Aleksandrov, G. Plunien, and V. M. Shabaev, Momentum distribution of particles created in space-time-dependent colliding laser pulses, Phys. Rev. D 96, 076006 (2017).

[108] I. A. Aleksandrov, G. Plunien, and V. M. Shabaev, Dynamically assisted Schwinger effect beyond the spatially-uniformfield approximation, Phys. Rev. D 97, 116001 (2018).

[109] C. Kohlfürst, Effect of time-dependent inhomogeneous magnetic fields on the particle momentum spectrum in electron-positron pair production, Phys. Rev. D 101, 096003 (2020).
[110] J. Ambjorn, J. Greensite, and C. Peterson, The axial anomaly and the lattice Dirac sea, Nucl. Phys. B 221, 381 (1983).

[111] H. Lehmann, K. Symanzik, and W. Zimmermann, Zur Formulierung quantisierter Feldtheorien, Nuovo Cim. 1, 205 (1955).

[112] K. Tharmalingam, Optical absorption in the presence of a uniform field, Phys. Rev. 130, 2204 (1963).

[113] J. Callaway, Optical absorption in an electric field, Phys. Rev. 130, 549 (1963).

[114] P. Copinger, K. Fukushima, and S. Pu, Axial Ward Identity and the Schwinger Mechanism: - Applications to the Real-Time Chiral Magnetic Effect and Condensates, Phys. Rev. Lett. 121, 261602 (2018).

[115] H. J. Warringa, Dynamics of the chiral magnetic effect in a weak magnetic field, Phys. Rev. D 86, 085029 (2012).

[116] U. Heinz, Thermalization at RHIC, in IX Hadron Physics and VII Relativistic Aspects of Nuclear Physics: A Joint Meeting on QCD and QCP, 28 March - 3 April 2004, Rio de Janeiro, edited by M. Bracco, M. Chiapparini, E. Ferreira, and T. Kodama, AIP Conf. Proc. No. 739 (AIP, New York, 2004), p. 163.

[117] P. Bozek and I. Wyskiel-Piekarska, Indications of early thermalization in relativistic heavy-ion collisions, Phys. Rev. C 83, 024910 (2011).

[118] M. Cardona, Modulation Spectroscopy, Solid State Physics, Suppl. 11, edited by F. Seitz, D. Turnbull, and H. Ehrenreich (Academic, New York, 1969).

[119] C. Kohlfurst, H. Gies, and R. Alkofer, Effective Mass Signatures in Multiphoton Pair Production, Phys. Rev. Lett. 112, 050402 (2014).

[120] G. R. Mocken, M. Ruf, C. Muller, and C. H. Keitel, Nonperturbative multiphoton electron-positron-pair creation in laser fields, Phys. Rev. A 81, 022122 (2010).

[121] T. Heinzl, A. Ildertonb, and M. Marklund, Finite size effects in stimulated laser pair production, Phys. Lett. B 692, 250 (2010).

[122] I. Akal, S. Villalba-Chavez, and C. Muller, Electron-positron pair production in a bifrequent oscillating electric field, Phys. Rev. D 90, 113004 (2014).

[123] C. Kohlfurst, Spin-states in multiphoton pair production for circularly polarized light, Phys. Rev. D 99, 096017 (2019).

[124] A. Wollert, H. Bauke, and C. H. Keitel Spin polarized electronpositron pair production via elliptical polarized laser fields, Phys. Rev. D 91, 125026 (2015).

[125] H. Taya, Mutual assistance between the Schwinger mechanism and the dynamical Casimir effect, arXiv:2003.12061. 